\title{
From Narrow-Gap and Semimagnetic Semiconductors to Spintronics and Topological Matter: A Life with Spins
}

\begin{abstract}
T. DIETL*
International Research Centre MagTop, Institute of Physics, Polish Academy of Sciences, Aleja Lotnikow 32/46, PL-02668 Warsaw, Poland

Doi: 10.12693/APhysPolA.139.355

*e-mail: dietl@MagTop.ifpan.edu.pl

The abundance of semiconductors in our smartphones, computers, fiber optic junctions, cars, light sources, photovoltaic and thermoelectric cells results from the possibilities of controlling their properties through doping, lighting, and applying various fields. This paper, a part of the volume celebrating 100 years of the Polish Physical Society, presents a biased selection of worthwhile results obtained by researchers at the Institute of Physics, Polish Academy of Sciences relevant, as seen today, to topological matter and spintronics. Comprehensive studies, combining materials development, experimental investigations, and theoretical description of narrow-gap and dilute-magnetic semiconductors have been especially significant in this context. This survey also emphasizes, in an autobiographical tone, a half of a century of the author's intellectual emotions accompanying the rise of ideas and quantitative theories, allowing identifying the physics behind ongoing and future observations.
\end{abstract}

topics: dilute magnetic semiconductors, spintronics, topological materials

\section{Introduction}

In my recorded talk [1], I presented recent breakthroughs in spintronic and topologicalmatter research relevant to today's and tomorrow's information-communication technologies. Such a program was addressed to broad audience of Polish Physical Society meetings - teachers, publishers, colleagues from different fields. Not surprisingly, therefore, given the special and ceremonial character of the meeting, Dariusz Wasik, the session chair, and the semiconductor community fellow, commented on the talk: "... as we are at the meeting of Polish physicists, I would like to underline their contributions and yours, Tomasz, to semimagnetic semiconductors and spintronics." Hence, this paper is an addendum to the presentation [1], and describes, in an ahistorical and self-centered way, a couple of past accomplishments of researchers at the Institute of Physics, Polish Academy of Sciences (IF PAN) concerning narrow-gap and dilute magnetic semiconductors relevant, as seen today, to topological matter and spintronics.

In a sense, this survey is complementary to a broad overview of semiconductor research in Poland presented by Maria Kamińska from the University of Warsaw's (UW) viewpoint [2], and also to Jan Gaj's contribution to a previous meeting of the Polish Physical Society [3], as well as to recollection reviews by Robert Gałąka (1937-2021) [4] and Andrzej Kisiel et al. [5]. Much systematized information can be found in a recent unsurpassed monumental masterpiece of Andrzej Wróblewski [6]. Obituaries and reminiscences emphasize the importance of our Masters and colleagues, Leonard Sosnowski (1911-1986) [7], Jerzy Mycielski (1930-1986) [8, 9], Witold Giriat (1926-2001) [10, 11], Jan Blinowski (1939-2002) [12, 13], Jan Gaj (1943-2011) [14], and Michał Nawrocki (1943-2018) [15]. I have been unable to find a history of the Department of Solid State Physics in Zabrze, specializing in II-V narrow-gap and dilute magnetic semiconductors, run for years by Lidia (1925-1996) and Witold (1923-2008, Gulag prisoner and Monte Cassino hero) Żdanowicz. Also, there is no adequate account of the Unipress contribution to the physics of narrow-gap semiconductors, InSb in particular.

I hope that the present overview will trigger a series of papers presenting the topic from different angles or describing the developments of other branches of physics in a similar way. This article purposely presents only references to papers with the IF PAN affiliation. The one hundred reviews I am co-author of, particularly the two most recent ones [16, 17], contain extensive and, hopefully, well-balanced citations of contributions from other institutions. 
More specifically, Sect. 2 presents the issue of surface and bulk Dirac cones in topological and narrowgap semiconductors. The emergence of semimagnetic semiconductors is described in Sect. 3. Afterwards, Sect. 4 contains a discussion about the origin of positive and negative magnetoresistances in solids, whereas Sect. 5 is devoted to the influence of resonant states on carrier mobility. Section 6 presents studies on quantum structures of dilute magnetic semiconductors. Sections 7, 8, and 9 are devoted to properties, mechanisms of spin-spin interactions, and the distribution of magnetic ions in dilute ferromagnetic semiconductors, respectively. Finally, the search for topological superconductors is described in Sect. 10.

\section{Surface and bulk Dirac cones in narrow-gap semiconductors: from basement to attic}

I am not alone regarding the ARPES visualization of 2D topological surface Dirac states in $\mathrm{Pb}_{1-x} \mathrm{Mn}_{x}$ Se [18], as IF PAN's most significant accomplishment in the second decade of the 21st century. These studies were initiated by Tomasz Story immediately after a suggestion of the MIT/Northeastern group that SnTe-type materials belong to a class of topological crystalline insulators (TCIs), just discovered theoretically by Liang $\mathrm{Fu}$ at MIT. The Warsaw/Lund/Stockholm results were not only submitted earlier than those of Sendai/Osaka and Princeton/Shanghai/ Berkely/Boston/Villigen/Huston/Tampei collaborations but also showed the full gapless Dirac cones, not only their valence band part and, moreover, traced the topological phase transition in a single sample. Furthermore, Ryszard Buczko's tight-binding computations supported the photoemission data, and nicely illustrated the presence of surface gapless cones only if the band structure is inverted, i.e., anion $p$-states reside above cation ones. Not surprisingly, therefore, this publication is already among ten most cited IF PAN's papers, and Tomasz Story was invited to speak about it at many venues, including the 32nd International Conference on the Physics of Semiconductors (ICPS; Austin TX 2014) and APS March Meeting (San Antonio TX 2015). For these studies, Andrzej Szczerbakow obtained high-quality $\mathrm{Pb}_{1-x} \mathrm{Mn}_{x}$ Se single crystals by a $20 \mathrm{k} €$ self-selecting vapor growth method [19], perfected during his retainment times in a basement just below five ultrahigh vacuum equipment for MBE, sometimes referred to as mega backs' epitaxy. Similarly to the case of topological insulators of $\mathrm{Bi}$ am Sb chalcogenides developed at Princeton by Robert Cava's group, the IV-VI program at IF PAN had been carried on in the pre-topological epoch with thermoelectric applications in mind.

Experimental and theoretical studies of TCIs are continued in IF PAN on many fronts and also in a worldwide collaboration. Among others, Matthias
Bode's STM group in Würzburg discovered on surfaces of Szczerbakow's $\mathrm{Pb}_{1-x} \mathrm{Mn}_{x}$ Se, $1 \mathrm{D}$ topological Dirac cones adjacent to odd surface step edges [20], presumably, the first observation of higher-order topological states. The present studies of topological surface bands, making use of wonderfully working Kraków's SOLARIS ARPES facilities, include Weyl semimetals [21] and ferromagnetic TCIs [22], also examined from a theoretical perspective [23, 24].

The case of TCIs, topological insulators, and earlier of graphene, shows that electron Dirac cones have been with material physicists for a long time. But when and where have they been observed for the first time? This question brings us back to the beginning of the 1960s and to the attic of UW's physics building at 69 Hoża Street. There, IF PAN's Witold Giriat initiated the growth of $\mathrm{HgTe}$ under the wings of Leonard Sosnowski, the Master and Mentor of many generations of Warsaw's semiconductor physicists, who, while in the UK elucidated the physics of $p-n$ junctions (three Nature articles, 1946-1947), and was elected as the President of the International Union of Pure and Applied Physics (IUPAP) for the term 1978-1981. From today's perspective, IF PAN studies of narrow-gap semiconductors brought several worthy accomplishments relevant to present works on topological matter of semiconductors and semimetals.

\subsection{Inverted band structure of $\mathrm{HgTe}$}

Sylwester Porowski and co-workers developed at IF PAN high-pressure technique (the first step on Sylwester's heroic way to found at PAS the prominent Institute of High-Pressure Physics, Unipress). Measurements of the Hall resistance and the Seebeck coefficient $\alpha$ up $1.2 \mathrm{GPa}$ at room temperature demonstrated that $\mathrm{HgTe}$ has an inverted band structure [25], just proposed by Steven Groves and William Paul at Harvard for gray tin. Robert Gałązka, under the supervision of Leonard Sosnowski, grew bulk $n$ - $\mathrm{Hg}_{0.9} \mathrm{Cd}_{0.1} \mathrm{Te}$ and, by measuring $\alpha$ in high magnetic fields, determined the values of the effective mass $m^{*}$ of electrons as a function of their concentration $n$ [26]. The results pointed to $m^{*} \propto n^{1 / 3}$, proving a linear (Dirac) dispersion, $E(k)=v_{f} k$. Amazingly, the resulting Fermi velocity $v_{f}=1.1 \times 10^{8} \mathrm{~cm} / \mathrm{s}$ is within $10 \%$ of the graphene value.

Despite the lack of topological protection, finetuning of the energy gap to the Dirac point by hydrostatic pressure made it possible to observe, a dozen years later, electron mobility as high as $2 \times 10^{7} \mathrm{~cm}^{2} /(\mathrm{V} \mathrm{s})$ in $\mathrm{Hg}_{0.94} \mathrm{Mn}_{0.06} \mathrm{Te}$ [27] (see Sect. 5). The thermoelectric measurements of $\mathrm{HgTe}$ (and afterward of HgSe) were taken over and sophisticated by Andrzej Jędrzejczak, my MSc supervisor (1972/1973), whose data, together with mine, I put to our first publications [28, 29]. Andrzej shared my wife Maniela's and my happiness on our son Marek's birth, as his godfather. 
About that time, Andrzej Mycielski and Lucjan Sniadower studied optical properties of a $\mathrm{HgTe}-$ based system, which brought Andrzej to discovering, together with Jacek Baranowski (UW), the infrared detecting capabilities of $\mathrm{Hg}_{1-x} \mathrm{Cd}_{x} \mathrm{Te}$. Andrzej did not wait long to start supplying these detectors around, for instance, to mines as local heat sensors. Józef Piotrowski took over and developed this technology in the Military University of Technology, and founded Vigo System, now a stock-exchange global company located in Warsaw's area, which produces top-level infrared detectors. This company is one of MagTop's industrial partners.

\subsection{Electron transport phenomena}

The quantitative description of the above experimental data was carried out making use of Jerzy Kołodziejczak and Leonard Sosnowski's theory of transport phenomena in semiconductors with a non-parabolic band structure proposed for InSb by Evan Kane in his famous 1957 paper completed at General Electric. One of the key concepts introduced by them at that time was the energy-dependent momentum effective mass, $m^{*}=\hbar k / v=\hbar^{2} k(\mathrm{~d} \varepsilon / \mathrm{d} k)^{-1}$. Leonard Sosnowski presented emerging IF PAN's experimental and theoretical results in his invited talks at the 7 th and 9th ICPS (Paris 1964, Moscow 1968). Jerzy Ginter and Wanda Szymańska were also members of the team, soon enriched by the dynamism of Włodzimierz Zawadzki. The crucial development, inspired by Henry Ehrenreich (1928-2008), then at General Electric and later at Harvard, was the use of proper Kohn-Luttinger amplitudes $u_{\boldsymbol{k}}$ that took under adequate consideration the $\boldsymbol{k}$-dependent spinmomentum locking [30]. This formalism, summarized by Włodzimierz in his plenary talk at the 11th ICPS (Warsaw 1972) and in a comprehensive Adv. Phys. paper [31], became a world standard in describing momentum, energy, and spin relaxation in narrow-gap semiconductors, InSb in particular. $\mathrm{PhD}$ students Piotr Bogusławski and me, in daily collaboration with Wanda Szymańska, generalized this theory to zero-gap semiconductors [32, 33]. That theory I converted to a comprehensive code which was successfully used for quantitative descriptions of my and others' electron mobility and thermomagnetic data taken over a wide temperature range and - as we would say now - across the topological phase transition in $\mathrm{Hg}_{1-x} \mathrm{Cd}_{x}$ Se [34-36] and $\mathrm{Hg}_{1-x} \mathrm{Cd}_{x} \mathrm{Te}$ [37].

\subsection{Relativistic effects and semirelativity}

As demonstrated in the last decade, topologically non-trivial states live at the boundaries of materials with inverted band structures, such as $\mathrm{Hg}-$, Bi-, and Sn-based chalcogenides and cadmium arsenide. Relativistic effects, such as the spinorbit interaction and the velocity-mass correction, stand behind the pertinent properties of topological matter, including spin-locking and the appearance of inverted band structures ( $p$-type anion orbitals above $s$ or even $p$ cation states) in materials containing heavy elements. This band inversion, quantified by topological invariances, results in gapless boundary states when stitching wave functions at interfaces between materials belonging to different topological classes. At the same time, effective (multiband) Hamiltonians explaining quasi-particles properties in solids, particularly of electrons in narrow-gap semiconductors and semimetals, are often formally identical to various forms of equations put forward by Paul Dirac (1902-1984), Hermann Weyl (1885-1955), Ettore Majorana (1906-unknown), and Frank Wilczek to describe existing or hypothetical elementary particles, fermions in particular.

While the first report about an analogy between the four-band model (including spin) equations for narrow-gap semiconductors and the Dirac equation goes back to Leonid Keldysh (1931-2016), since 1964 Włodzimierz Zawadzki has persistently developed and extended this analogy [38], also in the reverse direction, for instance, by deriving a formula for the spin magnetic moment of relativistic electrons [39]. In general, however, the four-band version of the Kohn-Luttinger $k p$ theory does not suffice to describe semiconductor properties quantitatively. Accordingly, eight or fourteen band variants were developed at MIT, in Paris, IF PAN, and other labs in order to tackle the magneto-optical and magneto-transport phenomena in these systems.

\subsection{Quantized magnetic fields}

Warsaw's physicists have often been taking their samples with them and carried out charge transport and optical measurements in quantized magnetic fields in various labs around the globe. However, helium started to be available (Jerzy Raułuszkiewicz, 1927-2005, took care of it), and cryostats with superconducting coils were purchased in the beginning of the 1970s, when we moved to a new IF PAN campus on aleja Lotników in 1973. Andrzej Mycielski with a group of $\mathrm{PhD}$ students, Małgorzata Dobrowolska among them, developed an infrared magnetooptical lab, while me with Daniel Dobrowolski and gifted technician Piotr Siemiński (1947-1984) — a setup for measurements of the Shubnikov-de Haas (SdH) oscillations employing a field-modulation technique [40].

Simultaneously, Daniel wrote a code providing Landau level energies within the Pigeon-Brown model, a key tool for the interpretation of magnetooptical and magnetotransport data. Our results, published in conference proceedings, have had a rather limited impact. That was also the fate of a report on the theoretical and experimental demonstration that a large difference between Dingle and mobility temperatures did not originate from samples inhomogeneities but could be accurately explained in terms of the quantum lifetime [41], the 
fact rediscovered at IBM a couple of years later. However, the built setups and codes, together with the already existing growth facilities, turned out to be essential for a quick take off with studies on semimagnetic semiconductors or, according to the terminology imposed in the US, dilute magnetic semiconductors (DMSs).

\section{Emergence of semimagnetic semiconductors}

There are two events integrating semiconductor physicists in Poland: Friday seminars at UW, started by Leonard Sosnowski after his return from the UK in 1948, and Jaszowiec annual meetings initiated by Witold Giriat, whose 12th and 49th editions were postponed because of the martial law and the pandemic situation in 1982 and 2020, respectively. During one of the first Friday seminars I attended, Robert Gałązka, following his return from Jacek Furdyna's lab at Purdue, unfolded a vision of studying mainstream semiconductor compounds with group II or group III cations substituted partly by transition metal ions [42]. Thus, these new systems - referred to as semimagnetic semiconductors — should show some remarkable properties of magnetic semiconductors but without long-range order (hence the name semimagnetic). At the same time, owing to the host's excellent semiconductor characteristics, they should be more accessible for investigating, understanding, and applying.

Within this program, Jacek Kossut at IF PAN, but under the supervision of Jerzy Mycielski at UW, began investigating theoretically electron scattering by magnetic impurities in narrow-gap semiconductors [43, 44], Anna Pajaczkowska established that typically up to $50 \%$ percent of $\mathrm{Mn}$ could be introduced to II-VI compounds preserving excellent structural characteristics [45], Andrzej Mycielski elaborated a method of Mn purification, and transferred the invention to his on-campus company (now PUREMAT Technologies, an industrial partner of MagTop) that has became the world leader in supplying pure Mn to research labs. Barbara Witkowska, an electrical engineer and then growth technology expert, has assisted Andrzej for half a century. Ursula Dębska (1939-2020) (later at Purdue) and Andrzej Szczerbakow joined the growth team.

Although busy with writing my $\mathrm{PhD}$ thesis and associated papers [33-36], I was aware of the approaching wave, signalized by a striking behavior of $\mathrm{SdH}$ oscillations in $\mathrm{Hg}_{1-x} \mathrm{Mn}_{x} \mathrm{Te}$ [46], giant Faraday rotation in $\mathrm{Cd}_{1-x} \mathrm{Mn}_{x}$ Te found at UW by Jan Gaj and Michał Nawrocki [47] (soon commercialized in optical isolators by TOKIN company in the Sendai region), and Gérald Bastard and co-workers' magnetooptical data obtained for $\mathrm{Hg}_{1-x} \mathrm{Mn}_{x}$ Te at ENS in Paris [48, 49]. I also attended Robert Gałąka's invited talk at the 14th ICPS in Edinburgh, August 1978, in which those results made an extraordinary impact on the audience [50]. At the same time (1977 and 1978), Sergiy Ryabchenko's Kiev group reported giant exchange exciton splittings in CdTe:Mn and ZnTe:Mn, respectively.

After completing my $\mathrm{PhD}$, to build expertise, on Robert's recommendation, I spent 10 months in 1978 at an École Polytechnique lab founded by Ionel Solomon (1929-2015), which in collaboration and competition with physicists centered around Boris Zakharchenya (1928-2005) at Ioffe, made pioneering contributions to spintronics associated with spin-orbit and hyperfine interactions in semiconductors. Despite the fact that a decade has elapsed, May 1968 was still in the air. Georges Lampel, the discoverer of spin pumping in semiconductors, as a protest against the incarceration of physicist Yuri Orlov and mathematician Natan Sharansky declined to be an editor of the reference book Optical Orientation (North Holland 1984) in the series containing chapters written alternately by Western and Soviet physicists. At the same time, École Polytechnique elite students demanded supplying L'Humanité (indirectly supported by Soviets) to the Palaiseau campus newspaper stands. On arrival, Ionel - my Master - showed me our working horse, an automatized setup for resistivity measurements of hydrogenated amorphous silicon, equipped with Commodore, the first personal computer I had ever seen. Nevertheless, we all participated in a 45 min protest (making me a strike expert in the Solidarity carnival times), when a secretary did not obtain an increase in salary after refusing to attend a computer course.

By field-effect measurements, we showed that surface depletion layers control the film properties [51]. I think my main accomplishment was to invalidate, by a series of experiments, Ionel's idea that the Staebler-Wronski effect, killing photovoltaic cell's performance, is a surface phenomenon. Amorphous silicon expertise introduced me to the physics of disordered (see Sect. 4) and 2D systems (see Sect. 6). At the same time, reading for a change older and newer local PhD theses, I became familiar with the fascinating world of spin-dependent phenomena in solids. At Paris dinners, with my distant cousins Andrzej and Jerzy Mycielski (visiting Claudette Rigaux's lab) as well as with Gérald Bastard, Yves Guldner, and Jan Gaj (staying in the Claude Benoit à la Guillaume, 1925-1994, group), the bright prospects of DMSs became clear to me.

Arriving back in Warsaw, with my MSc student Marcin Otto, I built the first Warsaw's setup for magnetization measurements of DMSs, an a.c. susceptometer. We did not find any effects of electron concentration changes by annealing in $\mathrm{Hg}_{1-x} \mathrm{Mn}_{x}$ Se, but provided supplementary information for Margaret's magnetooptical data [52] and, in parallel to Robert's SQUID measurements at Purdue, detected spin-glass freezing in $\mathrm{Hg}_{1-x} \mathrm{Mn}_{x} \mathrm{Te}$, the results published somewhat later with Faraday rotation data [53]. The magnetization behavior in $n$ - $\mathrm{Cd}_{1-x} \mathrm{Mn}_{x}$ Se was essential 
in building up the understanding of the physics of bound magnetic polarons [54]. To learn more about how to change carrier density by the field effect, I spent July 1980 in the mecca of 2D semiconductor systems — Frederick Koch (1937-2012) lab at the Technical University of Munich, where I heard Klaus von Klitzing announcing, perhaps for the first time, his discovery of the quantum Hall effect [55]. I also spoke there about DMSs, and both of us were recommended by Fred to Noboru Miura as invited speakers at the Oji International Seminar on High Magnetic Fields in Semiconductor Physics in Hakone, September 1980, a satellite event to the 15th ICPS in Kyoto, where Jan Gaj also delivered an invited talk on DMSs.

With Fujiyama in the window, I presented an overview of Warsaw/Paris findings, adopting the $s-d$ Vonsovskii model to (II, Mn) VI compounds and pointing out that $p-d$ hybridization accounts for exchange coupling of $\Gamma_{8}$ carriers to Mn spins, the suggestion confirmed theoretically by Anadi Bhattacharjee et al. in Orsay.

I would single out five durable accomplishments of studies devoted to bulk DMSs, partly described in early reviews $[56,57]$ and given below in the further subsections.

\section{1. $s p-d$ exchange energies}

In order to interpret the inter-band magnetooptics data on $n$ - $\mathrm{Hg}_{1-x} \mathrm{Mn}_{x}$ Te, Gérald Bastard, with the support of Jerzy Mycielski, described the influence of $\mathrm{Mn}$ ions on the effective mass electrons in terms of the molecular-field and virtual-crystal approximations [48, 49], an approach taken over by Jacek Kossut to interpret Marek Jaczyński's SdH results [46] and by Gaj, Ginter, Gałąka (the $3 \mathrm{G}$ model), who assumed that to quantify giant $s p-d$ exchange splitting of free exciton states, the Landau quantization can be neglected in wide-band gap semiconductors [58]. These models, with minor modifications, have served for decades to describe magnetooptical and magnetotransport results and, thus, to determine the $s-d$ and $p-d$ exchange integrals (denoted as $\alpha$ and $\beta$, respectively) in a dozen of II-VI compounds containing cationsubstitutional $\mathrm{Mn}$ ions but also $\mathrm{Cr}, \mathrm{Fe}$, and Co ions. This activity was carried on by the Warsaw/Paris collaboration and, somewhat later, primarily by Andrzej Twardowski and co-workers at UW, Jacek Furdyna's group at Purdue/Nore Dame, Donald Heiman et al. at MIT, Shojiro Takeyama and co-workers in Himeji and Tokyo, and other teams. Ab initio computations by Henry Ehrenreich group at Harvard and Alex Zunger et al. in Golden CO, as well as a tight-binding approach put forward by Jan Blinowski (UW) and Perła Kacman (IF PAN) allowed us to understand the signs and magnitudes of $\alpha$ and $\beta$ [59].

A similar approach [60] has been, at least semiquantitatively, successful in describing magnetooptical splittings in $\mathrm{PbTe}$ and $\mathrm{PbSe}$ with substitutional Mn and Eu cations [61] and the EPR Knight shift in the of Mn in PbTe and SnTe [62]. We continue this line of research extending the theory for arbitrary $\boldsymbol{k}[63]$.

\subsection{Strong coupling effects}

Attempts to go beyond virtual-crystal and molecular-field approximations have a long history, often put forward in the context of the so-called mismatched alloys. The key insight, which I share, is that, similarly to the Kondo effect and superconductivity, perturbation approaches come short, as a substantial modification of the local potential means that a bound state can be formed, even for the substitution by the element with the same valence, such as $\mathrm{N}$ in GaAs, $\mathrm{Mn}$ in $\mathrm{ZnO}$, or Fe in GaN. A non-perturbative Wigner-Seitz-like approach to the $p-d$ exchange interaction that grows up with decreasing the anion-cation bond length was proposed by Claude Benoit à la Guillaume and Denis Scalbert, to which I incorporated the non-magnetic alloy potential [64]. Our work explained a mysterious increase of the exchange energy $\left|N_{0} \beta\right|$ with lowering $x$ observed by exciton magnetospectroscopy for $\mathrm{Cd}_{1-x} \mathrm{Mn}_{x} \mathrm{~S}$.

In a series of his excellent works, Jakub Tworzydło at UW summed up a class of diagrams describing self-energy of holes at the top of the valence band in the presence of randomly distributed Mn spins of arbitrary magnetization, and demonstrated the quantitative accuracy of our model for $\mathrm{Cd}_{1-x} \mathrm{Mn}_{x} \mathrm{~S}$. However, even more surprising was the outcome of magnetooptical studies carried out for $\mathrm{ZnO}$ and GaN doped with $\mathrm{Co}$ and $\mathrm{Mn}$ in mid2000 by Wojciech Pacuski and co-workers at UW, Grenoble, and IF PAN. The results implied the $N_{0} \beta$ values of the opposite sign and much reduced amplitude compared to those stemming from photoemission and XAS, as well as expected from the chemical trends.

I realized that Jakub's approach can be extended to the case, when the hole is bound to the transition metal ion, and showed that the model explains the reversal of the valence band splitting [65], in agreement with experimental data for $\mathrm{Ga}_{1-x} \mathrm{Fe}_{x} \mathrm{~N}$ [66] and $\mathrm{Zn}_{1-x} \mathrm{Mn}_{x} \mathrm{O}$ [67]. I think that splitting of the conduction band into two branches observed in mismatch alloys could be explained by Jakub's theory generalized for $k=0$. Somewhat related is the issue of atypical magnitudes of $\alpha$ reported for certain DMSs. For instance, David Awschalom's group found negative values of $\alpha$ for paramagnetic $\mathrm{Ga}_{1-x} \mathrm{Mn}_{x}$ As with $x \leq 0.13 \%$, which I assigned to the exchange interaction with the hole residing on the Mn acceptor, the suggestion wonderfully quantified by Cezary Śliwa [68].

\subsection{Bound magnetic polarons}

Do effective electrons affect Mn spins? Around 1980, the effects of bound magnetic polarons (BMPs) have been uncovered optically by 
the Paris/Warsaw collaboration. Particularly clear were Michał Nawrocki and co-workers' spin-flip Raman scattering (SFRS) data for $n$ - $\mathrm{Cd}_{1-x} \mathrm{Mn}_{x}$ Se [69], which revealed spin splitting of donor electrons even in the absence of $\mathrm{Mn}$ macroscopic magnetization. In 1980/1981, Józef Spałek started coming from Kraków and brought to us his knowledge about magnetic semiconductors, particularly on theories of BMPs developed for those materials. It became clear to me that previous approaches missed the key ingredient the significance of thermodynamic magnetization fluctuations in the case of localized electrons.

To resolve the central-spin problem, as we would say now, I learned the Ginzburg-Landau approach to phase transitions from the book of Shangkeng Ma (1940-1983), and formulated the question in terms of functional integrals without the "box" approximation [70, 71]. With Józef, we nicely interpreted Michał's SFRS and my magnetization results [54]. Here, we were faster than the MIT group around Peter Wolff (1923-2013), and soon the Dietl-Spałek model, yielding the shift, width, and shape of the spin-flip line has been verified by a dozen of groups, also in the context of widely studied excitonic magnetic polarons (EMPs). Later, Tomasz Wojtowicz, using Witold Plesiewicz's homemade SQUID, confirmed quantitatively our predictions concerning BMP magnetization [72].

My works on BMPs have earned me international recognition as a theoretician. In that hat, I interpreted with the Günther Bauer group photomagnetization data [73] and later, while in Grenoble, results on the formation time of EMPs obtained by my hosts and previously at Brown, MIT, and IBM [74]. At the same time, I was rightly sceptical about the idea of the free magnetic polaron appearing in the DMS literature at that time. More recently, in 2015, I confirmed that the central spin problem can be solved semiclassically, i.e., I reproduced accurately within our formalism the recent results, obtained by solving the quantum Liouville equation for the whole system or from quantum Monte Carlo numerical simulations, concerning the dynamics of the localized electron spin in the hyperfine field of nuclear magnetic moments [75].

\subsection{Antiferromagnetic superexchange}

There is a consensus that the exchange interaction between dilute $\mathrm{Mn}$ ions in II-VI and IV-VI semiconductors is bilinear in spin operators and antiferromagnetic for all distances between magnetic ions [59]. The Hamiltonian contains the scalar Heisenberg and the pseudo-dipole terms and, depending on the spin pair symmetry in a given host, non-scalar contributions, such as the Dzyaloshinskii-Moriya component, visible clearly in EPR studies. As suggested by Józef Spałek et al. [76], and shown quantitatively by the Ehrenreich group at Harvard, the short-range superexchange is the dominant interaction mechanism in Mn-based II-VI DMSs. A linear dependence of the CurieWeiss temperature on the Curie constant in the high temperature limit [76] proved the uncorrelated distribution of $\mathrm{Mn}$ ions and also Co ions [77]. Inelastic neutron and light scattering, together with steps in $M(H)$ dependencies in high magnetic fields, have provided quantitative information on exchange energies for $\mathrm{Mn}, \mathrm{Co}$, and Eu nearestneighbor pairs in II-VI and IV-VI DMSs [78]. Lowtemperature specific heat and magnetization measurements carried out in various labs across the globe allowed establishing that freezing temperature $T_{\mathrm{f}} \propto x^{p}$, where $p=1.3$ for $\left(\mathrm{Cd}_{1-x} \mathrm{Mn}_{x}\right)_{3} \mathrm{As}_{2}$ and $p=2.3$ for wide band-gap Mn-based DMSs [4]. The Faraday rotation [79] and quantum noise [80] served to determine the character of spin-glass dynamics.

3.5. Playing with magnetism in magnetically doped $\mathrm{Pb}_{1-x} \mathrm{Sn}_{x} \mathrm{Te}$

Two pioneering works of Tomasz Story and the co-authors demonstrated that it is possible, by changing carrier density, to trigger ferromagnetism in $\mathrm{Pb}_{1-x-y} \mathrm{Sn} y \mathrm{Mn}_{x} \mathrm{Te}$ [81] and to alter the strength of antiferromagnetic coupling in $\mathrm{Sn}_{1-x} \mathrm{Gd}_{x} \mathrm{Te}$ [82]. In the former case, the intrinsic antiferromagnetic interaction of $\mathrm{Mn}$ ions is overcompensated by carrier-mediated RKKY-type ferromagnetic coupling once the holes start to occupy twelve side $\Sigma$ valleys, as revealed by magnetization studies under hydrostatic pressure [83]. In the case of $\mathrm{Sn}_{1-x} \mathrm{Gd}_{x} \mathrm{Te}, \mathrm{Gd}$ ions introduce occupied donor and empty acceptor states $\left(5 d^{1}\right.$ and $5 d^{2}$, respectively), which are resonant with the valence band. By annealing, one decreases the concentration of native acceptors, which shifts the Fermi energy toward the $5 d^{2}$ level. According to the data and the model put forward in [82], antiferromagnetic coupling between Gd spins becomes then resonantly enhanced. In those systems, vacancy-related hole densities attain the level of $7 \times 10^{20} \mathrm{~cm}^{-3}$, not reachable in the case of II-VI DMSs but available in $\mathrm{Ga}_{1-x} \mathrm{Mn}_{x} \mathrm{As}$, where $\mathrm{Mn}$ ions act as acceptors providing holes to a relatively simple and well-known valence band (see Sect. 7). As already mentioned (see Sect. 2), those materials combine magnetism with topological characteristics, and are in the center of MagTop studies.

\section{Why do spin effects account for positive magnetoresistance, whereas orbital phenomena - for negative, and not vice versa?}

"What about spin?" - I asked Philip Anderson (1923-2020) to break the embarrassing silence after his plenary talk at Montpellier's IUPAP 16th ICPS in 1982 [84]. In his lecture, he presented the Gang of Four work on quantum localization (inspired by David Thouless) and corresponding 
millikelvin results taken at Bell Labs. He jumped on the topic as, according to his later writings, he spent last evenings then discussing to what extent spin physics could account for the apparent disagreement between the theory and data on the quantum metal-to-insulator transition (MIT) in Si:P. My perspective was different: in IF PAN, we just began millikelvin measurements of $\mathrm{Hg}_{1-x} \mathrm{Mn}_{x} \mathrm{Te}$ [27] (see Sect. 5) and magnetoresistance studies of $\mathrm{Cd}_{1-x} \mathrm{Mn}_{x}$ Se [85] and $p-\mathrm{Hg}_{1-x} \mathrm{Mn}_{x} \mathrm{Te}$ [86] in the vicinity of the MIT.

The textbook paradigm was that the Lorentz force results in a positive magnetoresistance (MR), whereas spin-disorder scattering (especially in the Kondo limit) and bound magnetic polarons make MR negative in dilute magnetic materials and magnetic semiconductors. In the case of $n-\mathrm{Cd}_{1-x} \mathrm{Mn}_{x} \mathrm{Se}$, it was apparently the other way round: the weak-field MR was negative in $n$-CdSe and positive in paramagnetic $n$ - $\mathrm{Cd}_{0.95} \mathrm{Mn}_{0.05} \mathrm{Se}[85]$ ! Immersed in a summer scenery of southern France, so bright after a long martial-law darkness in Soviet-controlled Poland, I realized that DMSs (see Sect. 3) once more opened new research horizons - this time to test fundamentally new theoretical predictions concerning the crucial role of subtle interference effects in one-electron and many-body Anderson-Mott localization in disordered systems, put forward by Boris Altshuler, Arkadii Aronov (1939-1994), Hidetoshi Fukuyama, David Khmelnitskii, Patrick Lee, T.V. Ramakrishnan, and others, the understanding accelerated by a Soviet-US joint meeting at Armenian Lake Sevan in September 1979. When I learned that Jacek Kossut would host Yoshi Ono from the University of Tokyo, I asked them to consider MR of DMSs caused by quantum localization. Their paper considering the one-electron case [87] was a good starting point to develop a more comprehensive numerical code, the task taken over by Maciej Sawicki.

Importantly, we had in place already at that time the bulk DMS growth technology (developed first under the leadership of Witold Giriat and then of Robert Gałązka and Andrzej Mycielski, see Sect. 3), and a dilution fridge program (initiated in IF PAN by Lucjan Śniadower and Piotr Sękowski, who then emigrated to France and Germany, respectively), and taken over in 1982 by highly talented self-made cryogenic expert Witold Plesiewicz and me. Witold, till 2010, fabricated about 70 helium and nitrogen cryostats, distributed over entire Poland, but also exported to, e.g., University of Tokyo and Zürich's ETH. He was my wedding witness, we never missed any illegal Solidarity demonstration (dressed in running shoes), and sequentially hosted in our apartments the Editorial Office of the underground Jan Strękowski's "Tygodnik Wojenny" (War Weekly) appearing in the years 1982-1985 and later the recording studio of the Solidarity Program II radio. Actually, our most active IF PAN colleague and future
Senate member Zbigniew Romaszewski was sentenced to four and a half years in prison for organizing the first Solidarity radio. Zbigniew and his wife Zofia were among those whose struggle resulted in the falling walls of 1989. Zbigniew's Moscow visit as an IF PAN delegate was in fact a cover for the famous meeting between KOR and Andrei Sakharov in 1979 .

There were four new accomplishments collected in PhD theses of Tomasz Wojtowicz (1988), Maciej Sawicki (1990), Jan Jaroszyński (1990), and Paweł Głód (1995), made possible by their contribution to the development of millikelvin measurement setups:

1. Effect of spin splitting. The giant $s-d$ exchange splitting produces a field-dependent mass in the diffusion and Cooperon poles in quantum conductivity corrections brought about by electron-electron interactions, which leads to a sizable positive $\mathrm{MR}$ on the conducting side of the MIT. The effect was initially found and interpreted quantitatively in $n-\mathrm{Cd}_{1-x} \mathrm{Mn}_{x} \operatorname{Se}: \operatorname{In}$ [88], and later investigated in various DMSs around the world, but also in IF PAN on epilayers from various labs, $n-\mathrm{Zn}_{1-x} \mathrm{Mn}_{x} \mathrm{O}: \mathrm{Al}$ [89], $n-\mathrm{Zn}_{1-x} \mathrm{Co}_{x} \mathrm{O}: \mathrm{Al} \quad[90], \quad \mathrm{Cd}_{1-x} \mathrm{Mn}_{x} \mathrm{Te}$ HEMTs [91], and $n-\mathrm{Ga}_{1-x} \mathrm{Mn}_{x} \mathrm{~N}: \mathrm{Si}$ [92]. At the same time, somewhat surprisingly to many, effects of spin-disorder scattering upon many-body quantum localization are not relevant in paramagnetic DMSs, as temperature at which thermal broadening $k_{\mathrm{B}} T$ becomes smaller than spin-disorder scattering rate corresponds to the onset of carrier-driven ferromagnetic ordering of localized spins.

2. Temperature-dependent localization. We discovered an abrupt conductivity drop below $1 \mathrm{~K}$ accompanied by a negative colossal MR (CMR) in the close vicinity of the MIT in $n-\mathrm{Cd}_{1-x} \mathrm{Mn}_{x}$ Se $[88,93,94]$. To make a long story short: the key notion here is the disorder-driven spatial separation into regions with larger and smaller carrier density near the MIT, which - owing to carrier-mediated ferromagnetic coupling between Mn - results in mesoscopic magnetization fluctuations leading to quantum localization [91, 95]. Thus, the physics behind the phenomenon is similar to that put forward by Elbio Dagotto, Adriana Moreo and others as well as Eduard Nagaev (1934-2002) to explain the origin of CMR in ferromagnetic oxides and semiconductors in the vicinity of $T_{\mathrm{C}}$. Thus, within this model, critical carrier scattering and the associated negative $\mathrm{CMR}$ originate rather from spatial fluctuations of $T_{\mathrm{C}}$ in the MIT neighborhood than from thermodynamic magnetization fluctuations at $T_{\mathrm{C}}$ (which is below $100 \mathrm{mK}$ in the studied $n$-type DMSs). 
3. Critical behavior. It was realized by Steve von Molnár at IBM and by us that while quantitative modeling of conductance and, in particular, of negative CMR is not yet possible across the MIT, the presence of the fieldinduced insulator-to-metal transition in appropriately selected samples allows one to determine critical exponents of the AndersonMott quantum transition, a much debated issue at that time. Especially attractive was the case of $p-\mathrm{Hg}_{1-x} \mathrm{Mn}_{x} \mathrm{Te}$, in which, as shown by Jerzy Mycielski, an increase of pd exchange splitting makes the localization length to be more and more determined by the $\Gamma_{8}$ light hole mass. This effect strongly enhances CMR, and allowed us to study the critical behavior of resistance, resistance anisotropy, Hall effect, dielectric constant, and hopping length [96-98]. I called the Landau Institute and asked Alexander Finkelstein to provide us with dynamic renormalization group equations for the spin-polarized case, which we compared to experimental data [96], the procedure later followed by many groups for the MIT in various disordered systems.

4. Rashba weak antilocalization. We were the first to reveal the influence of the Rashba spin-orbit term on transport phenomena experimentally by observing a tiny positive MR (weak antilocalization - WAL) in $n$-CdSe:In below $1 \mathrm{~K}$, which was taken over above $20 \mathrm{mT}$ by a stronger negative MR - a manifestation of the Aharonov-Bohm-type of interferences for electrons diffusing in disordered media (weak localization MR) [88]. Notably, the determined magnitude of the Rashba parameter was in excellent agreement with the value known from the strength of electron spin-resonance in $n$ - $\mathrm{Cd}_{1-x} \mathrm{Mn}_{x}$ Se, studied experimentally by Jacek Furdyna's and Denis Drew's groups, and theoretically by Peter Wolff at MIT. The WAL MR was even smaller, but detectable, in $n$ - $\mathrm{ZnO}: \mathrm{Al}$ [89] and $n$-GaN:Si [99]. At the same time, our data allowed verifying, presumably for the first time quantitatively, the theory of the phase coherence time, confirming the dominant role of carrier-carrier scattering at low temperatures [88].

Innovative and comprehensive nature of this research resulted in inviting me to speak at IUPAP's 18th International Conference on Low Temperature Physics, Kyoto 1987 [100] and 19th ICPS, Warsaw 1988 [101], as well as at the Meetings of the Condensed Matter Division of EPS, Budapest 1988 and of APS, St. Louis MO 1989. At that time, we carried out with Donald Heiman millikelvin spin-flip Raman scattering measurements at the MIT Francis Bitter Magnet Lab, unsuccessfully looking for BMPs on the metal side of MIT in $n-\mathrm{Cd}_{1-x} \mathrm{Mn}_{x} \mathrm{Se}$ [102]. On those occasions, and with the help of Stephan von Molnár (1935-2020), I made two seminar tournées over a dozen US universities and research centers (including Bell Labs and IBM) traveling between them on Greyhound buses. It was quite a progress as compared to my lowest-level construction jobs in London and Brussels suburbs which provided funds for seven hitchhiking summer tours between youth hostels and homes of my beloved relatives living in several magnificent cities in western and southern Europe (1967-1974; 25,000 km).

For my 40th birthday, in 1990, I obtained a professor title. I was also asked to chair the International Conference on Electron Localization and Quantum Transport. The conference was organized in Jaszowiec with the logistic help of Elżbieta Zipper at Silesian University but we arranged a banquet near Kraków in a spacious grotto $100 \mathrm{~m}$ below the ground, a part of the medieval Wieliczka Salt Mine complex — the UNESCO World Heritage Site. One of the scheduled highlights was an after-dinner talk to be given by Nevill Mott (1905-1996) [103] about his Goettingen times with Max Born. Sir Nevill, however, passed away that night, canceling a couple of days earlier his trip to Poland because of a flu infection.

There have been many interesting research followups. For instance, I found that surprising negative MR of ferromagnetic $p$ - $\mathrm{Ga}_{1-x} \mathrm{Mn}_{x}$ As persisting up to at least $27 \mathrm{~T}$ at $T \ll T_{\mathrm{C}}$ [104] can be quantitatively interpreted in terms of the orbital single-electron WL interference effect [105, 106], as the exchange band splitting is field-independent at low temperatures and also precludes the WAL's MR appearance in ferromagnets. This insight was confirmed by comprehensive MR studies of $p$-Ga $\mathrm{Ga}_{1-x} \mathrm{Mn}_{x}$ As carried out by Dieter Weiss's group in Regensburg, the weak-field data showing, additionally, the presence of demagnetization effects and field-dependent magnon scattering at non-zero temperatures. Another line of recent studies concerns ferromagnetic and non-magnetic topological semiconductors which exhibit negative WL MR and positive WAL MR, respectively (see, e.g. [107]).

Finally, I have to note that despite all those developments there have been systematic assignments of negative MR and/or non-linearities in the Hall resistance to spin-disorder scattering by the existing magnetic impurities or by hypothetical spins (or two-level systems) on defects, prior to checking the role of orbital WL MR, whose magnitude can be evaluated without any adjustable parameters.

\section{How do resonant states enhance electron mobility?}

The question on whether and under which conditions localized and extended states can co-exist at the same energy appears in many contexts, and is also central to the condensed matter physics, as 
it concerns, for instance, the survival of magnetic moments in metals and the issue of disorder-driven metal-insulator transition. The key notion is resonant scattering that dramatically lowers conductivity of metals containing impurities if they give rise to quasi-localized states with binding energies near the Fermi energy. Not surprisingly, this question was addressed for acceptors in zero-gap semiconductors, such as HgTe, for which Boris Gelmont and Mikhail Dyakonov predicted in 1972 a larger binding energy than lifetime broadening. This created an abundance of theories providing the strength of resonant scattering, a prediction apparently confirmed by a minimum in the $\mathrm{HgTe}$ conductance at temperatures at which the Fermi energy assumes the expected positions of acceptors states, always present in real materials.

However, this insight was called into question by Władysław Walukiewicz who showed in a qualitative model that optical phonon scattering between the conduction and valence bands might account for the electron conductivity minimum [108]. Our rather comprehensive eight band $\mathrm{kp}$ theory of electron transport in zero- and narrow-gap zincblende semiconductors, developed with Wanda Szymańska employing a variational solution of the Boltzmann equation [33] and verified for HgSe [34] and $\mathrm{Hg}_{1-x} \mathrm{Cd}_{x}$ Se [35], confirmed Władysław's suggestion by showing good agreement of the theory and experimental data for $\mathrm{Hg}_{1-x} \mathrm{Cd}_{x} \mathrm{Te}$ as a function of temperature and $x$ with no adjustable parameters [37, 109].

So, are resonant states irrelevant in semiconductors? Absolutely not — they can lead to a significant... enhancement of carrier mobility. To describe magnetic impurities in metals, Anderson considered a competition between intra-site correlation energy $U$ and hybridization between impurity and band state $V_{\boldsymbol{k} d}$. In the case of resonant states in semiconductors, I argued $[100,110]$ that a competition of $V_{\boldsymbol{k} d}$ with intersite Coulomb energy $E_{\mathrm{C}}$ is essential. If the width of the EfrosShklovskii gap produced by $E_{\mathrm{C}}$ is larger than the tunneling rate from the resonant state to the band, the efficiency of resonant scattering will be much reduced. Moreover, as noted already in 1983 by the Nikolaj Brandt (1923-2015) group at Moscow University, who investigated Witold Giriat's zero-gap donor-compensated $p$ - $\mathrm{Hg}_{1-x} \mathrm{Mn}_{x} \mathrm{Te}$ and by Jerzy Mycielski in the context of Andrzej Mycielski's HgSe:Fe [111], $E_{\mathrm{C}}$ results in a correlated spatial arrangement of charges on resonant impurities, which dramatically enhances electron mobility and reduces the Dingle broadening at low temperatures. This model explains quantitatively the mobility magnitudes in HgSe:Fe in which $\mathrm{Fe}^{3+} / \mathrm{Fe}^{2+}$ donor states re-side $0.2 \mathrm{eV}$ above the conduction band bottom [110, 112], elucidates the mechanism leading to mobility as high as $20 \times 10^{6} \mathrm{~cm}^{2} /(\mathrm{V} \mathrm{s})$ found in pressurized $\mathrm{Hg}_{0.94} \mathrm{Mn}_{0.06} \mathrm{Te}$ [27], and explains the pressure dependence of electron mobility in n-GaAs, in which $\mathrm{Si}$ donors form DX resonant states [113]. Amazingly, under modulation doping conditions, the barrier donors constitute resonant states for the channel carriers. According to the Vladimir Umansky et al. 2008 paper (Rehovot/Stuttgart collaboration), the correlated arrangement of donor charges is essential to achieve electron mobility as high as $35 \times 10^{6} \mathrm{~cm}^{2} /(\mathrm{V} \mathrm{s})$ in GaAs HEMT structures.

Jacek Kossut was invited to present IF PAN results at the 20th ICPS, Thessaloniki 1990. On one evening of the conference, I participated in a gala dinner in an elegant beach hotel. We sat at exactly the same illuminated dining tables I saw with jealousy in my eyes from behind a fence a year earlier when we ate plain cooked noodles in front of our little tent. A family dinner in that restaurant would then cost my IF PAN monthly salary even though our 10 years old daughter Zosia gave up the tour with us preferring to take advantage of her last chance to visit East Germany with her dance group.

\section{Quantum structures of dilute magnetic semiconductors}

Shortly after winning independence, Poland joined CERN in 1991. Other initiatives got a boost, too. In 1992, Robert Gałązka and Jacek Kossut, IF PAN's Director and Deputy Director, respectively, founded an MBE program, with Jacek Kossut and Tomasz Wojtowicz as persons in charge. In 1993, they made operating a new MBE Lab equipped with the first commercial MBE system in Poland, purchased from the EPI company in the USA, using IF PAN own funds. Simultaneously, government subsidies financed a couple of research groups interested in low-dimensional systems. Jan Gaj coordinated the project, and Manijeh Razeghi, a pioneer in epitaxial techniques for semiconductors, chaired a panel of external advisors. This initiative made it possible to develop, with a significant involvement of Grzegorz Karczewski and Elżbieta Janik, the MBE growth technology of II-VI compounds.

At the same time Jerzy Wróbel and I, in collaboration with Eliana Kamińska and Anna Piotrowska at the Institute of Electron Technology, constructed a clean room at IF PAN equipped with electron beam lithography and auxiliary nanofabrication tools. They were funded by other grants, including Austrian Ost-West program we applied to with Günther Bauer with whom many other IF PAN colleagues and I had a long record of friendly and efficient collaboration. Jerzy's proficiency is best illustrated by his Stern-Gerlach solid-state spin-filter whose fabrication required five electronolithography levels and lift-off of four different metals [114]. It seems that he triggered the use of micromagnets by the spin-qubit community. IF PAN's epitaxy and nanofabrication capabilities have been enriched by Tomasz Wojciechowski and Tomasz Wojtowicz's decades-long tireless activities, culminating with 
the opening in 2017 of the new Laboratory of Technological Processes of Semiconductor Nanostructures and Devices, now further expanding within the MagTop project.

Of course, the acquiring of funds for the quantum structure program was facilitated by our earlier accomplishments in the physics of low-dimensional systems $[115,116]$. For instance, my then $\mathrm{PhD}$ student Grzegorz Grabecki had started in 1980 fabricating field-effect transistors of $p-\mathrm{Hg}_{1-x} \mathrm{Mn}_{x} \mathrm{Te}$, and successfully demonstrated the influence of the $s p-d$ exchange interaction on the $\mathrm{SdH}$ oscillations of interfacial electrons as a function of the electric field and temperature [117]. My assignment of some strange data to inversion layers at grain boundary defects in $p$ - $\mathrm{Hg}_{1-x} \mathrm{Mn}_{x}$ Te [118] allowed Grzegorz to show that the anomalous Hall effect, expected in the presence of magnetic ions, does not affect the precision of Hall resistance quantization [119, 120]. At the same time, Marian Herman (1936-2015) [121] and Janusz Sadowski were constructing an MBE setup for IV-VI compounds.

In 1987, Gorbachev decided to open boarders with Poland, closed in 1981. I immediately went to Moscow and Leningrad, where over an aromatic Armenian-style coffee in a tiny apartment inhabited by the Boris Altshuler family, my appetite to study mesoscopic phenomena in DMS spin-glasses grew even further. We did not continue that discussion when Boris revisited me during Warsaw's ICPS a year later, as without any fear he used the time to ask other guests, Eva Andrei among them, where it was better to work, in Europe or in the US?

Undaunted by this, taking advantage of a relatively long mean free path of electrons in narrowgap semiconductors we started to study mesoscopic universal conductance fluctuations (UCFs) just employing photolithography. At that time, Andre Geim entered my office and, on seeing what was going on, immediately advised us to use oil from not too good pumps in our scanning electron microscope as a resist. He actually was faster than we [122], and reported already in 1990 mesoscopic spin-dependent UCFs as a function of the magnetic field in a GaAs nanowire. However, I argued somewhat later that Andre and co-workers observed actually SdH oscillations, not UCFs [123]. On hearing complaints coming from the $\mathrm{mK}$ lab that UCFs in our $n$ - $\mathrm{Cd}_{0.99} \mathrm{Mn}_{0.01} \mathrm{Te}: \mathrm{In}$ submicron wire show an atypical temperature dependence, I realized that UCFs in paramagnetic DMSs originate primarily from the redistribution of carriers between spin subbands, whereas both the Aharonov-Bohm-type of interferences and spin-disorder scattering are of lesser importance [123]. It took another year or two before Jan Jaroszyński took a wonderful set of data for $\mathrm{Cd}_{1-x} \mathrm{Mn}_{x}$ Te:I submicron wires in the spin-glass regime [80]. His analysis of the second spectra of conductance noise indicated that the Huse-Fisher droplet model applies to spin-glass freezing in random antiferromagnets.
There have been about 600 papers reporting results obtained on II-VI and later IV-VI quantum structures, often containing Mn, grown by IF PAN's MBEs. The results have constituted topics of invited talks at International Conferences on II-VI Compounds, and of Tomasz Wojtowicz's invited talk at the 34th ICPS, Montpellier 2018 and plenary talk at the 18th International Conference on MBE, Flagsta 2014. Some of the findings are reviewed in the book edited by Jacek Kossut and Jan Gaj [124] and in the book chapter co-authored by Aleksandr Kazakov and Tomasz Wojtowicz [125]. One example of numerous activity lines is the mesoscopic physics in submicron wires pattered of $n$-type doped DMS epilayers, as described above. Another research direction was exploring the suitability of self-organized quantum dots containing a single Mn ion as a quantum information carrier [126]. Also, nanowires showing giant exchange splittings of excitonic lines were obtained [127].

An important research topic has concerned modulation-doped DMS quantum wells, in which mysterious narrow peaks superimposed on beautiful QHE data let me propose that the quantum Hall ferromagnet (QHF) state is formed on crossings of Landau levels, imposed by the giant $s-d$ exchange spin splittings [128]. Long electron mean free path and giant exchange spin-splitting allowed the observation of Landau-Zener transitions between spin sublevels in a helical magnetic field produced by Dy microstripes [129]. Tomasz Wojtowicz's insistence in improving structures' quality made that the modulation-doped CdTe [130] and later $\mathrm{Cd}_{1-x} \mathrm{Mn}_{x} \mathrm{Te}$ [131] quantum wells belong to an exclusive club of systems in which the fractional QHE has been observed. Those accomplishments constitute a starting point of MagTop struggle aiming at using domains of the QHF in both QHE and FQHE regimes as a platform hosting Majorana and, possibly, non-Abelian excitations, as proposed in papers co-authored by MagTop researchers [132, 133].

The acquired proficiency in ultra high-vacuum techniques was a good starting point to initiate partner collaboration between IF PAN and the highly successful PREVAC company, located in south-western Poland, and specializing in fabricating custom-designed ultra-high vacuum systems, such as MBE, ARPES, XPS, and others. This collaboration is continued and reinforced by MagTop, as PREVAC is one of MagTop's industrial partners.

\section{Saga of dilute ferromagnetic semiconductors}

In November 2020, my wife Maniela got a text message from her nephew congratulating us on the fact that her support and my instance made me the most cited scientist working now in Poland. The ensemble of papers on dilute ferromagnetic semiconductors contributed mostly to such a position in the ranking completed according to a methodology 
elaborated by Stanford/SciTech Strategies/Elsevier bibliometric experts. (Surely, other methodologies would reshuffle the order). Also, the EPS Condensed Matter Division Europhysics Prize, which I received with David Awschalom and Hideo Ohno in 2005, and the 2006 Foundation for Polish Science Prize were awarded to me for my work on ferromagnetic semiconductors and semiconductor spintronics.

On those occasions and later at similar ceremonial events, it has been underlined that our work bridged broadly and fundamentally the two leading fields of condensed matter physics: semiconductor physics and magnetism. We made mainstream nonmagnetic semiconductors ferromagnetic, in which the virtues of magnetic, electronic, and photonic systems are combined. This intersection of magnetism, semiconductor physics, materials science, and localization physics has led to the discovery of new phenomena essential for new generations of spintronic devices, the initiation of a massive world-wide materials science search for magnetic semiconductors that resulted in the discovery of FeAs-based superconductors and variety of materials with ferromagnetic signatures that remain to be understood. Ferromagnetism in semiconductors has been essential in exploring new ideas and concepts, some of which - like spin injection from a ferromagnet, electrical magnetization manipulation, tunneling anisotropic MR, and spin-orbit torque - have already been transferred to ferromagnetic structures of metals or complex oxides, others like interplay of spin-orbit coupling and exchange spin splitting - to antiferromagnetic spintronics and research on topological matter. Accordingly, broadly understood spintronics has become one of the central themes of contemporary condensed matter physics and its ICT applications.

Two recent reviews describe findings in the field of dilute ferromagnetic semiconductors in more technical terms and in a context of worldwide research [16, 17]. IF PAN's main accomplishments, achieved in collaboration with Grenoble, Linz, and Sendai teams, but also with Athens, Nottingham, Würzburg, and other centers, include:

1. Predicting $T_{\mathrm{C}}$. To establish conditions allowing for carriers'-mediated ferromagnetic ordering and to evaluate the magnitudes of Curie temperature $T_{\mathrm{C}}$ and of various thermodynamic quantities, I made use of all that broad knowledge gathered over decades for the mainstream semiconductor compounds, i.e., III-V and II-VI tetrahedrally bonded semiconductors with carriers residing at the Brillouin zone center b[134-136]. While in Grenoble (altogether 7 months in 1994-1997), stimulated by Yves Merle d'Aubigné (1933-2000), I demonstrated the equivalence of the RKKY and Zener models, and predicted $T_{\mathrm{C}}$ for various dimensionality structures of II-VI compounds in the mean-field approximation [134, 137]. Zener's model (Phys. Rev. B 81, 440 (1951)) in this context means competition between spin entropy and carrier energy lowering coming from exchange splitting of the band. The theoretically expected $T_{\mathrm{C}}$ s values were soon confirmed experimentally, particularly in the case of modulation doped $p-\mathrm{Cd}_{1-x} \mathrm{Mn}_{x} \mathrm{Te}: \mathrm{N}$ quantum wells [138, 139] (we understood the lack of hysteresis much later [140, 141]), $p$ - $\mathrm{Zn}_{1-x} \mathrm{Mn}_{x} \mathrm{Te}: \mathrm{N}$ epilayers brought to IF PAN in 1998 for magnetic and transport characterization by their developers, then PhD students, David Ferrand and Alberta Bonanni [142, 143], and in IF PAN's bulk $p$ - $\mathrm{Zn}_{1-x} \mathrm{Mn}_{x}$ Te:N [144, 145]. Notably, the predicted absence of ferromagnetism above $1 \mathrm{~K}$ in $n$-type samples was corroborated by millikelvin studies of $n-\mathrm{Zn}_{1-x} \mathrm{Mn}_{x} \mathrm{O}$ : $\mathrm{Al}$ [146]. Actually, much more impacting was my evaluation of $T_{\mathrm{C} S}$, in the mean-field approximation and within the $p-d$ Zener model, for a broad range of $p^{+}$-type tetrahedrally coordinated semiconductors: the theory was in agreement with existing data (notably for $\mathrm{Ga}_{1-x} \mathrm{Mn}_{x} \mathrm{As}$ ) and implied a survival of ferromagnetism to the above room temperature in gallium nitride, zinc oxide, and diamond containing $5 \%$ of $\mathrm{Mn}$ and $3.5 \times 10^{20}$ valence band holes per $\mathrm{cm}^{3}[135,136]$. So far, no such itinerant hole densities were achieved in the latter systems, but the theory was quantitatively verified for $\mathrm{Ga}_{1-x} \mathrm{Mn}_{x} \mathrm{As}, \quad \mathrm{Ga}_{1-x} \mathrm{Mn}_{x} \mathrm{P}, \quad \mathrm{In}_{1-x} \mathrm{Mn}_{x} \mathrm{As}$, $\mathrm{Ga}_{1-x} \mathrm{Mn}_{x} \mathrm{Sb}$, and $\mathrm{In}_{1-x} \mathrm{Mn}_{x} \mathrm{Sb}$ [147], in which $\mathrm{Mn}$ acceptor radius is large enough to allow for hole delocalization. Also, pressure studies are consistent with model [148, 149]. Other thermodynamic properties, such as magnetization $M(T, H)$ and specific heat are also well described by the $p-d$ Zener model, provided that corrections to the mean-field theory are included [150, 151].

To put the understanding of carriermediated ferromagnetism in semiconductors in a broader context, it is worth contrasting it with another approach, in which the understanding often just means the identification of an $a b$ initio code or an exchange-correlation potential that provides the magnetic ground state observed experimentally.

2. Micromagnetic properties. I think it is hard to over-value the beauty and power of micromagnetic theory which, together with the Landau-Lifshitz-Gilbert equation, describe magnificent magnetization patterns and their dynamics in ever more striking magnetic materials. But what about material input parameters with which simulations are run? Typically, we rely on experimental determination, as only recently $a b$ initio computations can provide an estimate of the 
magnetic anisotropy energy (MAE) of, say, bulk iron or a dilute ferromagnetic semiconductor $\mathrm{Ge}_{1-x} \mathrm{Mn}_{x} \mathrm{Te}$ [152]. In contrast, our $p$-d Zener model, built with the spinorbit interaction taken into account, provided MAE magnitudes as a function of strain, temperature, hole and Mn concentrations in $\mathrm{Ga}_{1-x} \mathrm{Mn}_{x}$ As $[135,136]$. Combining that with the exchange stiffness theory developed by Allan McDonald's group, we were able, for instance, to describe stripe domains in $\mathrm{Ga}_{1-x} \mathrm{Mn}_{x}$ As films with perpendicular magnetic anisotropy [153]. Furthermore, the theoretically predicted change in MAE sign as a function of carrier density [136] was confirmed experimentally and explained in terms of the carrier redistribution between hole subbands [154]. Of course, specific materials science issues, such as anisotropic spinodal decomposition (see Sect. 9) made the physics of MAE much richer $[155,156]$ than it could be anticipated. Also, the presence of a superparamagnetic component, brought about by the proximity to the MIT, plays a role [157, 158]. Results of micromagnetic studies of $\mathrm{Ga}_{1-x} \mathrm{Mn}_{x}$ As and related ferromagnets by dozen groups world-wide (including our own $[158,159])$ show how far we have arrived with the understanding of these systems.

3. Functionalities. No doubt, the prospect of applications have been driving research in semiconductor spintronics. It was clear to me that the demonstration of changing the magnetic phase by light in modulation-doped $p$ - $\mathrm{Cd}_{1-x} \mathrm{Mn}_{x} \mathrm{Te}$ quantum well meant that other means, such as gating, would also provide a high degree of control over magnetic properties [138], a dream made real in the pin diode configuration in 2002 , i.e., five years later [139]. In the meantime, a series of ingenious works on magnetization manipulation by an electric field in gated $\mathrm{In}_{1-x} \mathrm{Mn}_{x}$ As and $\mathrm{Ga}_{1-x} \mathrm{Mn}_{x}$ As epilayers, a major step towards energy efficient magnetization switching, carried out by Hideo Ohno and co-workers (with me as a discussion partner while at Tohoku) at the turn of the millennium, shook the magnetic and semiconductors communities.

A contribution of IF PAN's researchers to this line of research was twofold. First, Maciej Sawicki constructed in Sendai and fully exploited a SQUID setup allowing measuring directly gate-induced magnetization changes [157, 160]. Second, I developed a theory describing $T C$ for systems with non-uniform carrier density [157], which successfully described the dependence of $T_{\mathrm{C}}$ on the gate voltage in a range of $\mathrm{Ga}_{1-x} \mathrm{Mn}_{x} \mathrm{As}$ MOS FETs [161]. At the same time, Cezary Śliwa showed [159] that the $p-d$ Zener model can nicely explain changes in the easy axis direction generated by gating [160]. Another pioneering work done at Tohoku was the demonstration of magnetic wall displacement by an electric current without a magnetic field assistance, the domain velocity being accurately described theoretically with my contribution [162]. At the same time, in the frame of European projects, Piotr Sankowski et al. have been writing tightbinding codes for computing spin injection in Zener-Esaki diodes [163] and magnetoresitance and anisotropic magnetoresistance in magnetic tunnel junctions [164].

When at immigration kiosks, I quoted "conference physicist" as my profession in the years 2001-2005. My 66 invited talks over these five years included plenary lectures at EPS meetings (Budapest 2002, Prague 2004, Bern 2005), EMRS (Warsaw 2004), 27th ICPS (Flagstaff AR 2004), half-plenary at JEMS (Dresden 2003), and invited talks at Joint MMM Inter-Mag Conference (San Antonio TX 2001) and IUPAP's International Conference of Magnetism (Rome 2003). Similar visibility of David Awschalom and Hideo Ohno, together with influential writings presenting accomplishments, but also open questions (my contribution included [165] and [166]), resulted in a spread of spintronic research as well as in the support by high-profile projects on semiconductor spintronics. IF PAN was part of the Ohno ERATO funding and of E.C. collaborative FENIKS, AMORE, SPINOSA, NANOSPIN, and SemiSpinNet projects, as well as my ERC FunDMS Advanced Grant. This external funding was topped by the substantial support provided by government agencies and the non-public Foundation for Polish Science. Tomasz Wojtowicz, while with Jacek Furdyna at Notre Dame, contributed significantly to the DARPA project [167]. More recently, the EAgLE project (2013-2016), obtained in the frame of the E.C. REGPOT initiative supporting long-term visits, helped lift the window to antiferromagntic spintronics [168, 169]. This project also supported my travels to present a half-plenary talk at the ICM (Barcelona 2015) and an invited talk at the 33th ICPS (Beijing 2016), where I presented my view on the origin of spin-spin interactions in ferromagnetic topological semiconductors.

In this two-decades-long saga, incredibly unique was my one-year stay at Tohoku in 1999. There, logistics was entirely ensured by Maniela, I had no meetings and virtually no teaching, all administration duties were taken over by Fumihiro Matsukura, and I could enjoy inspiring business lunches with Hideo Ohno in a tinn French-style restaurant, sometimes with Sukekatsu Ushioda, the future IUPAP President, at the table. Under these unique conditions and having a strong background in the DMSs physics [57] and in the envelope function formalism [33, 34], I was in the position to 
identify properly (i) a minimal Hamiltonian suitable for describing carrier-mediated ferromagnetism in tetrahedrally coordinated $p$-type semiconductors; (ii) thermodynamic and micromagnetic quantities that theory can provide; (iii) relevant theory constrains, such as the metal-to-insulator transition, strong coupling, self-compensation, and solubility limits [135]. It appears that over that year I was able to describe theoretically and examine numerically a more comprehensive set of phenomena and materials [136] than a group of five brilliant postdocs (now Professors across Europe) supervised by Allan MacDonald in Austin and Lu Sham in San Diego. A well-known bane of many papers is the lack of values of the parameters used, making it impossible to check the results. Surprisingly, one of the referees of our PRB [136] recommended the removal of information on materials parameters I employed, apparently to make our work less substantial.

And at the end, a little too didactic illustration of the German proverb Übung macht den Meister. I got terrific Grenoble spectroscopic results on 2D ferromagnetism in $p$ - $\mathrm{Cd}_{1-x} \mathrm{Mn}_{x}$ Te quantum wells from Yves Merle d'Aubigné (1933-2000) in February 1997 while at a family ski-week in Günther Bauer's secondary house in Bad Ischl. Being busy with Warsaw's College of Science I could stay at Grenoble only one week in April. During that week, I managed to write down two manuscripts, this time without any help of IF PAN's English writing guru, Hanka Przybylińska: (i) the PRL on the observation of 2D carrier-mediated ferromagnetism [138], confirming nicely my a year-old theoretical prediction [134], and (ii) the Rapid Comm on controlling excitonic reflectivity by giant exchange spin splitting in DMS photonic superlattices [170], an extension of earlier Grenoble work, with my theoretical input, on non-magnetic photonic Bragg superlattices [171]. On returning to Warsaw, I came up to Ewa Skrzypczak (1929-2020) in Hoża Street to tell her that she was absolutely right on one summer day twenty-eight years ego. On that day, she, as a deputy dean, consented to my repeat exam session in September. Despite a crowd in front of her door, on seeing my top grades in maths and physics, and the failed lowest level English test, she decided to deliver to me a quarter of an hour tutorial on the significance of English proficiency in physics (although she might have not known that I had similar failures with French and Russian while in Poznań's Marcinek high school).

\section{Why $p$ - $d$ Zener model and superexchange, but neither double exchange nor Van Vleck mechanism?}

I do not recall who approached me over coffee after a conference talk and gave me a friendly advice: "Take it easy, do not treat that personally, be actually proud of it — for many people, the way to magnify their visibility is to challenge the principal paradigm of the time." Indeed, soon after the appearance of our 2000 Science paper [135], it has become fashionable to contest its two crucial and mutually related presumptions: it has been argued that (i) the holes in $\mathrm{Ga}_{1-x} \mathrm{Mn}_{x}$ As reside in an impurity band, not in the valence band; (ii) the ferromagnetism of this material should be described in terms of double exchange, not by the $p$ - $d$ Zener model. This situation motivated Tomás Jungwirth at Prague's IoP CzAS to assemble arguments against the paradigm shift, and to publish them with quite an impressive co-authors list [172].

As reviewed elsewhere [16], the trouble of models involving the presence of an impurity band is that all experiments designed to demonstrate directly its existence, especially photoemission and scanning tunneling spectroscopy, have failed in $\mathrm{Ga}_{1-x} \mathrm{Mn}_{x} \mathrm{As}$. Also, results of state-of-the-art $a b$ initio band structure computations, such as hybrid functionals and the dynamic mean-field approximation, have not shown any impurity band. The data pointed out to a few percent admixture of $\mathrm{Mn} d$ orbitals to the wave function of holes at the valence band top, just what is needed to explain the experimentally observed magnitudes of the valence band offset and the $p$ - $d$ exchange energy. At the same time, the temperature dependence of low temperature conductivity, the Seebeck coefficient, infrared conductivity integrated over frequency implied the hole mass of the value similar to GaAs. Last but not least, as mentioned above, thermodynamic and micromagnetic properties of $\mathrm{Ga}_{1-x} \mathrm{Mn}_{x} \mathrm{As}$, and also magnetic circular dichroism [136], can be described within the pd Zener model with no adjustable parameters - no one has reported an attempt to do that within competing models.

All that is not to say that the physics and materials science of dilute ferromagnetic semiconductors is straightforward. It was clear to me right from the beginning [135] that the Anderson-Mott localization and the associated non-uniformities of magnetization, together with important issues of solubility limits and self-compensation as well as of the transition to a strong coupling case with decreasing the lattice constant need to be addressed experimentally. For instance, in the MIT vicinity, the resistivity tensor's absolute values can hardy be described quantitatively, and often even qualitative understanding is challenging [173]. Furthermore, it is now known [65, 174], also due to an excellent series of works performed at UW by, among others, Agnieszka Wołoś and Marcin Zając in the Maria Kamińska and Andrzej Twardowski groups, respectively, that GaN:Mn is in a strong coupling limit $(p-d$ hybridization alone can bind a hole on the Mn ion), a conclusion consistent with parallel studies carried out at Schottky in Garching. This meant, disappointingly, that resulting mid-gap Mn acceptors would not introduce any valence band 
holes that might lead to room temperature ferromagnetism we had predicted [135]. Interestingly, however, Marcin Zajacc, after completing his PhD degree, moved to Robert Dwiliński's AMMONO (the company that made the IEEE Spectrum's cover story, the July 2010 issue), and introduced Mn as a trap of residual donor electrons, always present in GaN. Now, 1.5" semi-insulating GaN:Mn substrates are the flagship product of the AMMONO/Unipress company.

To understand the $\mathrm{Ga}_{1-x} \mathrm{Mn}_{x} \mathrm{~N}$ physics, especially fruitful has been the collaboration with Alberta Bonanni's group at Kepler University in Linz, where $\mathrm{Ga}_{1-x} \mathrm{Mn}_{x} \mathrm{~N}$ epilayers have been obtained by MOVPE. More recently, Detlef Hommel, now in Wrocław, has entered with his coworkers and MBE-grown films to the loop. Extensive characterization employing various photon, electron, and ion beams demonstrated world-top crystal quality, low electron concentration (measured in $\mathrm{GaN}$ ), and random Mn distribution with no spinodal decomposition up to $x=0.1$ [175-179]. Seeing low-temperature ferromagnetism of $\mathrm{Mn}^{3+}$ ions in these samples, I asked Jacek Majewski to dig up his tight-binding code that had served a decade earlier to predict ferromagnetic superexchange coupling between $\mathrm{Cr}^{2+}$ ions in II-VI compounds [180]. Combining it with Monte Carlo simulations, we obtained a successful description of $T_{\mathrm{c}}(x)[177,179]$.

Why, however, despite the fact that the Fermi energy is pinned by the $\mathrm{Mn}^{2+} / \mathrm{Mn}^{3+}$ impurity band, do we speak about superexchange, not about double-exchange invented also by Clarence Zener? The key reason is that double exchange requires certain delocalization of $d$ band carriers, the case of, for instance, CMR oxides. By contrast, in $\mathrm{Ga}_{1-x} \mathrm{Mn}_{x} \mathrm{~N}$, the dilution and the random Mn distribution together with a sizable Jahn-Teller effect and short localization radius in the strong coupling limit cause that we are far from the MIT, deeply in the strongly localized regime. In agreement with the expectation of the AndersonGoodenough-Kanamori superexchange theory, we observed Mn-Mn coupling to be ferromagnetic if a majority of $\mathrm{Mn}$ ions is in the $3+$ configuration. In contrast, antiferromagnetic interactions take over if $\mathrm{Mn}^{2+}$ prevail, the case of $\mathrm{Ga}_{1-x} \mathrm{Mn}_{x} \mathrm{~N}$ samples with a high concentration of compensating donors. In particular, at least so far, we have not found any $T_{\mathrm{c}}$ maximum at half filling, as expected within the double exchange scenario. The semiinsulating character of $\mathrm{Ga}_{1-x} \mathrm{Mn}_{x} \mathrm{~N}$ led me to suggest that the influence of an electric field on magnetic properties resulted from sample deformation by the inverse piezoelectric effect [181], not by an alternation of carrier density in the impurity band.

At the turn of the 1970s and 1980s, Gérald Bastard, the future honorary chair of the 34th ICPS (Montpellier 2018), worked with his coworkers to understand the magnetization behavior of $M(T, H)$ in Mn-doped and Fe-doped HgTe.
They proposed, in particular, that antiferromagnetic coupling between $\mathrm{Mn}$ ions results from the Bloembergen-Rowland (BR) mechanism, an interband analog of the RKKY interaction, which could be strong in the zero-gap (topological) $\mathrm{HgTe}$, the coupling also considered by me and others in Warsaw [182]. We now know that antiferromagnetic superexchange actually dominates [59] (see Sect. 3). In contrast, in the light of later studies of various Fe-based DMSs in several labs, the assignment of a linear field-dependence $M(H)$ to the Van Vleck paramagnetism was correct (Serre et al., Proceedings of the Linz Narrow-Gap Conference, Linz 1981). In the ground-breaking theory paper of the IoPChAS/Stanford/Tsinghua collaboration on the quantization of the anomalous Hall effect in ferromagnetic topological materials (Science 2010), the authors used the formula I derived to study the BR contribution in $p$ - $\mathrm{Ga}_{1-x} \mathrm{Mn}_{x}$ As [136] to claim that the BR mechanism, called by them the Van Vleck paramagnetism, accounts for ferromagnetic coupling between $\mathrm{Cr}$ spins in topological $\mathrm{Bi}_{2} \mathrm{Se}_{3}$. I think, looking at the results of $a b$ initio computations and experimental data for various transition metals, that - leaving aside the interaction's name - also in topological Cr- and V-doped Bi and Sb chalcogenides, it is not the BR mechanism but the superexchange that prevails, yet it comes with the ferromagnetic sign, just as in the case of $\mathrm{Mn}^{3+}$ ions in $\mathrm{GaN}$.

\section{How I killed semiconductor spintronics and why it had it coming}

Of course, this section title is a paraphrase of How I Killed Pluto and Why It Had It Coming - a marvelous book by Mike Brown. In 2003, I started to worry that our prediction about the room temperature ferromagnetism in wide-band gap DMSs was becoming too successful [183]: high $T_{\mathrm{C}}$ was reported for DMSs, in which no ferromagnetism was expected or even, one year later, for compounds nominally without any magnetic ions! Moreover, Maciej Sawicki's magnetic data [184] and some other group results pointed to the presence of magnetic precipitation. Thus, the crucial challenge became proper nanocharacterization, a demanding task, particularly considering that I [185] and Hiroshi KatayamaYoshida's group in Osaka realized that precipitates may assume the host crystal structure, driven by chemical spinodal nanodecomposition.

To go to the point: the advanced nanocharacterization protocols developed by Alberta Bonanni in Linz for $(\mathrm{Ga}, \mathrm{Fe}) \mathrm{N}[186,187]$, Shinji Kuroda in Tsukuba for $(\mathrm{Zn}, \mathrm{Cr}) \mathrm{Te}$ [188], and by others, together with results of SQUID magnetometry (that has reached the art level $[187,188])$ and a progress in using appropriate $a b$ initio tools, achieved in Osaka, Golden Colorado, and other places shed new light on the interplay of DMS materials science and magnetic properties [17, 191, 192]: 
1. Origin of high $T_{\mathrm{C}}$. Ferromagnetic-like features surviving up to above room temperature originate from the presence of nanoregions with a large density of the magnetic constituent and, thus, are characterized by a high spin ordering temperature. The interplay of attractive chemical forces between magnetic ions (driven by a contribution of the $d$ orbitals to bonding), entropy, and kinetic barriers determine the degree of non-uniformity in the magnetic ion distribution, i.e., an effective solubility limit, for given growth conditions and post-growth processing. The resulting spinodal nanodecomposition can have a character of chemical or crystallographic phase separation, in the latter case precipitation of metal nonoparticles sometimes occur. Unforseen contamination by magnetic nanoparticles can also be involved in specific cases. Uncompensated spins on surfaces of antiferromagnetic nanocrystals may contribute to magnetic response [90]. Furthermore, various couplings kinds between magnetized regions increase apparent superparamagnetic blocking temperature [77]. In general, however, magnetic hystereses are tilted with a minute, if any, remanent magnetization and coercivity. No clear correlation exists between $T_{\mathrm{C}}$ and the nominal concentration of magnetic impurities.

2. Controlling aggregation of magnetic ions. As demonstrated for $(\mathrm{Zn}, \mathrm{Cr}) \mathrm{Te}[188]$ and $(\mathrm{Ga}, \mathrm{Fe}) \mathrm{N}$ [187], the aggregation efficacy and, thus magnetic ordering, can be controlled by co-doping with shallow impurities that changes the charge state of magnetic ions and, thus, chemical forces between them [193]. In some cases, impurity complexes containing magnetic ions and shallow dopants appear, the case of ( $\mathrm{Ga}, \mathrm{Mn}) \mathrm{N}$ doped with $\mathrm{Mg}$, in which, as demonstrated by the Linz/Warsaw collaboration, clusters of $\mathrm{Mn}$ with one to three charged $\mathrm{Mg}$ acceptors bound to it account for magnetic and optical properties of $\mathrm{Mn}$ ions [194]. There is some relation here with a self-compensation effect predicted for (Ga,Mn)As by Jan Mašek and Frantisek Macá in Prague, and found experimentally by Berkeley/Notre Dame researchers, who unsuccessfully tried to raise $T_{\mathrm{C}}$ by increasing the concentration of $\mathrm{Mn}$ or Be acceptors. Their glorious Rutherford backscattering data demonstrated that to halt an energy increase associated with a decrease in density of electrons participating in bonding (i.e., hole formation), Mn ions displace to interstitial donor positions. In this location, they lower $T_{\mathrm{C}}$, as they couple antiferromagnetically to substitutional Mn ion [195]. A process of interstitials' removing by low-temperature annealing, which increases $T_{\mathrm{C}}$, was then quantitatively examined [196].
3. Anisotropic spinodal decomposition. At some point, it became clear to me that conditions at the growth front are essential for the resulting distribution of magnetic ions. For instance, the fact that chemical force was found attractive for $\mathrm{Fe}$ ions but repulsive in the case of Mn pairs on the GaN surface [197] explained why it was possible to grow $\mathrm{Ga}_{1-x} \mathrm{Mn}_{x} \mathrm{~N}$ epilayers with a random distribution of Mn ions. Another important consequence of this insight was the realization that the magnitude of attractive force at the surface is larger for the nearest neighbor $\mathrm{Mn}$ cation dimer residing along $\langle 1 \overline{1} 0\rangle$ direction compared to the $\langle 110\rangle$ arrangement, as there is no anion bonding the $\langle 1 \overline{1} 0\rangle$ pair on the (001) surface of the zinc-blende crystals [198]. A beautiful group theoretical analysis of crystals with $\langle 1 \overline{1} 0\rangle$ dimers was carried out by Cezary Śliwa. His evaluations showed that indeed the anisotropic spinodal decomposition can explain the origin and the magnitude of the in-plane uniaxial magnetic anisotropy hitherto mysterious but essential for functionalities of $\mathrm{Ga}_{1-x} \mathrm{Mn}_{x}$ As [198]. This symmetry breaking, i.e., nematicity, is particularly strong in $\operatorname{In}_{1-x} \mathrm{Fe}_{x} \mathrm{As}$ and was directly revealed by element sensitive nanocharacterization [199]. Since this discovery, I have been promoting the idea that the nematicity observed, for instance, in the quantum Hall effect, unconventional superconductors, and magnetic oxides originates in many cases from quenched anisotropic distribution of defects, impurities, or alloy components formed during the growth rather than from a spontaneous symmetry breaking in the many-body electronic subsystem cooled down to sufficiently low temperatures.

There has been more than two thousand papers claiming the discovery of high temperature ferromagnetism in semiconductors, oxides, various forms of carbon, topological matter, and most recently, in atomically thin $2 \mathrm{D}$ layers containing a low concentration of magnetic constituent (say, $x<10 \%$ ) or nominal without any magnetic ions, $x=0$. Considering the lack of follow up reports on spintronic functionalities at room temperature, it appears right to agree with Alex Zunger, who once remarked: "those who carefully characterize their samples and worry about the reproducibility deprive themselves of publications in high impact journals." However, taking into account foreseen fuctionalities of metal/semiconductor/magnet hybrids and prospects of self-organized growth of novel magnetic nanostructures [17, 191], as well as a steady progress in controlling properties of embedded magnetic nanocrystals [200], it is quite possible that studies of magnetic nanocomposites may return to many labs. And a somewhat related question: do we need high temperature uniform 
ferromagnetic or antiferromagnetic semiconductors for future spintronic devices? As mentioned in the previous section, spintronic functionalities discovered in dilute ferromagnetic semiconductors operate wonderfully at room temperature in ferromagnetic metals. We should not forget either that ferrite antennas of ferrimagnetic oxides have been with us for many decades. Nevertheless, similarly to the case of superconductors, we will not stop struggling to push operating temperature higher of, say, the quantum anomalous Hall effect in samples of topological ferromagnetic or antiferromagnetic semiconductors [201], considering foreseen applications in quantum metrology and possibly in topological quantum computing.

\section{Road to topological superconductivity}

It was a wonderful night. It happened to me during a carnival period in 1987. Suddenly, when sweeping the horizontal magnetic field at $30 \mathrm{mK}$, the $x y$ recorder's needle started moving down, and after a dozen of seconds up, reviling a strong diamagnetic signal from our home-made a.c. magnetometer [202]. That diamagnetism appeared when the Earth magnetic field was compensated by the field generated by a coil pair I had recommended to install around the millikevin cryostat. This finding confirmed the origin of the resistance drop below $0.5 \mathrm{~K}$, found by Maciej Sawicki during his night shift a couple of weeks earlier, once he had decided to decrease the current to an irrationally low value. I still remember the vibrating silence that fell in the Jaszowiec 1987 lecture hall when at the end of my talk Physics of semiconductors below $1 \mathrm{~K}$ [203], after describing quantum localization phenomena in Mn-based DMSs and the latest literature results on the fractional quantum Hall effect, I disclosed our discovery of superconductivity in zinc-blende Bridgman grown $\mathrm{Hg}_{1-x} \mathrm{Fe}_{x}$ Se.

A detailed analysis showed that untypically low magnitudes of critical currents and fields as well as specific dependencies of resistivity and magnetic susceptibility on temperature can rather nicely be explained by the presence of few superconducting precipitates and the proximity effect [204]. Actually, unusually high electron mobility in HgSe:Fe, described in Sect. 5, accounted for a considerable Cooper pair diffusion length in HgSe:Fe. Using electron microscopy we revealed indeed the presence of some inclusions but we were unable to determine their nature. After annealing in Se vapor, the disappearance of superconductivity suggested that $\mathrm{Hg}$ droplets might be involved [204]. Now I consider my not asking Andrzej Mycielski then to grow FeSe as the greatest shame in my scientific career.

In 1986, superconductivity features were found in PbTe-SnTe superlattices by Akihiro Ishida and coworkers at Shizuoka University and, over next two decades, in a broad range of topological and nontopological IV-VI superlattices and heterostructurs grown by Alexander Sipatov and co-workers in Kharkov since 1988. Prompted by these results, Evelyne Tang and Liang Fu at MIT proposed in 2014 a theory of superconductivity, whose essential ingredient was a flat band formed by a periodic arrangement of misfit dislocations in topological materials. In our lab, we found superconductivity in Kiev's bulk PbTe [205] and in IF PAN's bulk $\mathrm{Pb}_{0.63} \mathrm{Sn}_{0.37} \mathrm{Se}[22]$, presenting in both cases strong arguments that metallic $\mathrm{Pb}$ and $\mathrm{Sn}$ precipitates, respectively were a source of Cooper pairs. Somewhat related and also surprising were Andreev reflection results for $\mathrm{In} / \mathrm{PbTe}[206]$ and $\mathrm{In} / \mathrm{NbP}$ heterostructures [21], taken by Grzegorz Grabecki et al. at IF PAN. The former pointed to $T_{c}$ magnitudes higher than that of metallic In. In the simplest interpretation, the In diffusion into $\mathrm{PbTe}$ resulted in the formation of superconducting $\mathrm{Pb}$ precipitates [206]. In contrast, the $\mathrm{In} / \mathrm{NbP}$ data suggest the presence of a low-gap superconductivity, presumably appearing in the Weyl semimetal NbP, proximitized by the superconducting In [21].

Even more striking is the observation by several groups of Andreev-like spectra in topological semiconductors and semimetals, nominally without any superconductor, i.e., by making point contacts of normal (e.g., Ag, $\mathrm{Au}$ ) or even ferromagnetic (e.g., Co) metals. For instance, Grzegorz Mazur and Krzysztof Dybko, employing a brand new Triton 400 dry dilution refrigerator with a vector magnetic field, installed in our lab by Maciej Zgirski and Marek Foltyn, found such spectra in samples with silver paint contacts to diamagnetic $\mathrm{Pb}_{1-y} \mathrm{Sn}_{y} \mathrm{Te}$ as well as to paramagnetic or ferromagnetic $\mathrm{Pb}_{1-x-y} \mathrm{Mn}_{x} \mathrm{Sn}_{y}$ Te with $\mathrm{Sn}$ content $y$ corresponding to the topological crystalline insulator phase [22]. Is this phenomenon caused by hardly detectable residual precipitates of superconducting metals? Does interfacial topological superconductivity account for it, as suggested in virtually all publications? Could it originate from topological gap states at domain walls of a $1 \mathrm{D}$ carrier liquid at surface atomic steps, as proposed by Wojciech Brzezicki et al. [23]? If so, how to make the discovery useful for sensors and/or topological quantum computation? This is a set of questions addressed by researchers from 13 countries at the International Centre for Interfacing Magnetism and $\mathrm{Su}-$ perconductivity with Topological Matter MagTop, headed since 2017 by me and Tomasz Wojtowicz at IF PAN.

\section{Epilog}

In spring 2020, I was invited by young UW Professors Krzysztof Pachucki and Piotr Wasylczyk to one of on-line weekly discussion meetings they organize for a selected group of students. To start, I showed them viewgraphs with pieces of advice on how to run in the front, not behind the others (of the sort that publishing is essential but more important is uncovering a novel challenge, and solving it). However, the main advice was to realize 
that the research landscape is dynamic, and tips useful in one epoch may fail in another. For instance, I was fast in appreciating the role of arXiv, and applying in the first call for the KBN project and, later, for the E.C., Maestro, AdG ERC, and IRA projects. In turn, I was too late in abandoning meaningless titles, such as "Millikelvin Studies of Mixed-Valence HgSe:Fe" and conference proceedings (but surely one should move around, to be inspired by great people and wonders of the world). After consulting Andrzej Mycielski, I started my pre-MSc experimental work in 1971 under the supervision of Andrzej Jędrzejczak, who using his sophisticated thermoelectric setup, needed help in urgent collecting of the Nernst-Ettingshausen data for $n$-InSb, to be presented by Włodzimierz Zawadzki in his plenary talk at the 9th ICPS (Warsaw 1972).

In a lecture delivered at a meeting of Student Research Groups in Physics in Eódź that fall, I described my fascination, which survives until today, that a piece of a semiconductor is a laboratory, in which we can observe and quantify the flow of electrons under electric and magnetic fields. It was, however, a little later when I started to experience a passionate, not to say sensual, pleasure in that moment when the intricate puzzle turned into a clear picture - it could be the realization that magnetic hysteresis at $\mathrm{mK}$ temperatures is generated by a piece of type II superconductor used for wire's soldering: it could be the recognition that low field $\mathrm{SdH}$ oscillation in a $p$-type sample originates from a grain boundary in a nominally perfect single crystal; it could be the understanding that thermodynamic fluctuations or the inverse piezoelectric effect constitute the mechanisms explaining surprising optical or magnetization data. I think that such moments of illumination (to refer to Krzysztof Zanussi's film) have actually been the main driving force on my way.

\section{Acknowledgments}

I would like to thank my wonderful family (now enlarged by nine grandchildren) for continuous support and encouragement, and my past and present collaborators for the great time we have had together. The International Research Centre MagTop is funded by the Foundation for Polish Science through the IRA Programme financed by the EU within SG OP Programme.

\section{References}

[1] T. Dietl, Recorded talk presented at the Extraordinary Congress of Polish Physicists, 2020.

[2] M. Kamińska, Recorded talk presented at the Extraordinary Congress of Polish Physicists, 2020.

[3] J. Gaj, Post. Fiz. 45, 125 (1994) (in Polish).
[4] R.R. Gałązka, Phys. Status Solidi B 243, 759 (2006).

[5] A. Kisiel, B. Pukowska, M. ZimnalStarnawska, Działalność naukowa Zakładu Fizyki Ogólnej Instytutu Fizyki Uniwersytetu Jagiellońskiego, 2002 (in Polish).

[6] A.K. Wróblewski, Historia Fizyki w Polsce, PWN, 2020).

[7] M. Grynberg, M. Nawrocki, Post. Fiz. 53, 287 (2002) (in Polish).

[8] J. Blinowski, M. Grynberg, Post. Fiz. 37, 404 (1986) (in Polish).

[9] J.K. Furdyna, Phys. Today 39, 82 (1986).

[10] A. Mycielski, Post. Fiz. 52, 192 (2001) (in Polish).

[11] R. Brazis, Studium Vilnense A 12, 45 (2015) (in Polish).

[12] P. Kacman, Post. Fiz. 54, 213 (2003) (in Polish).

[13] Ł. Turski, Post. Fiz. 54, 214 (2003) (in Polish).

[14] M. Grynberg, M. Nawrocki, Post. Fiz. 62 , 89 (2011) (in Polish).

[15] J. Suffczyński, Post. Fiz. 70, 34 (2019) (in Polish).

[16] T. Dietl, H. Ohno, Rev. Mod. Phys. 86, 187 (2014).

[17] T. Dietl, K. Sato, T. Fukushima et al., Rev. Mod. Phys. 87, 1311 (2015).

[18] P. Dziawa, B.J. Kowalski, K. Dybko et al., Nat. Mater. 11, 1023 (2012).

[19] A. Szczerbakow, K. Durose, Progr. Cryst. Growth Charact. Mater. 51, 81 (2005).

[20] P. Sessi, D. Di Sante, A. Szczerbakow et al., Science 354, 1269 (2016).

[21] G. Grabecki, A. Dąbrowski, P. Iwanowski et al., Phys. Rev. B 101, 085113 (2020).

[22] G.P. Mazur, K. Dybko, A. Szczerbakow et al. Phys. Rev. B 100, 041408(R) (2019).

[23] W. Brzezicki, M.M. Wysokiński, T. Hyart, Phys. Rev. B 100, 121107 (2019).

[24] A. Łusakowski, P. Bogusławski, T. Story, Phys. Rev. B 103, 045202 (2021).

[25] R. Piotrzkowski, S. Porowski, Z. Dziuba, J. Ginter, W. Giriat, L. Sosnowski, Phys. Status Solidi B 8, K135 (1965).

[26] R.R. Gałązka, L. Sosnowski, Phys. Status Solidi B 20, 113 (1967).

[27] M. Sawicki, T. Dietl, W. Plesiewicz, P. Sękowski, L. Śniadower, M. Baj, L. Dmowski, in: Application of High Magnetic Fields in Semiconductor Physics, Ed. G. Landwehr, Springer, Berlin 1983, p. 382 . 
[28] T. Dietl, A. Jędrzejczak, Phys. Status Solidi B 71, K39 (1975).

[29] A. Jędrzejczak, T. Dietl, Phys. Status Solidi B 76, 737 (1976).

[30] W. Zawadzki, W. Szymańska, Phys. Status Solidi B 45, 415 (1971).

[31] W. Zawadzki, Adv. Phys. 23, 435 (1974).

[32] W. Szymańska, P. Bogusławski, W. Zawadzki, Phys. Status Solidi B 65, 641 (1974).

[33] W. Szymańska, T. Dietl, J. Phys. Chem. Solids 39, 1025 (1978).

[34] T. Dietl, W. Szymańska, J. Phys. Chem. Solids 39, 1041 (1978).

[35] R.J. Iwanowski, T. Dietl, W. Szymańska, J. Phys. Chem. Solids 39, 1059 (1978).

[36] R.J. Iwanowski, T. Dietl, J. Phys. C: Solid State Physics 11, 3239 (1978).

[37] J.J. Dubowski, T. Dietl, W. Szymańska, R.R. Gałązka, J. Phys. Chem. Solids 42, 351 (1981).

[38] W. Zawadzki, J. Phys.: Condensed Matter 29, 373004 (2017).

[39] W. Zawadzki, Phys. Rev. D 3, 1728 (1971).

[40] T. Dietl, P. Siemiński, W. Dobrowolski, "Układ do pomiarów efektu Szubnikowade Haasa", Miesięcznik Naukowo Techniczny: Pomiary, Automatyka, Kontrola 12, 421 (1976).

[41] T. Dietl, J. Phys. Colloques 39, C6-1081 (1978).

[42] R.R. Gałązka, Post. Fiz. 28, 601 (1977) (in Polish).

[43] J. Kossut, Phys. Status Solidi B 72, 359 (1975).

[44] J. Kossut, Phys. Status Solidi B 78, 537 (1976).

[45] A. Pajaczkowska, Prog. Crystal Growth Charact. 1, 289 (1978).

[46] M. Jaczyński, J. Kossut, R.R. Gałązka, Phys. Status Solidi B 88, 73 (1978).

[47] J.A. Gaj, R.R. Gałązka, M. Nawrocki, Solid State Commun. 25, 193 (1978).

[48] G. Bastard, C. Rigaux, A. Mycielski, Phys. Status Solidi B 79, 585 (1977).

[49] G. Bastard, C. Rigaux, Y. Guldner, J. Mycielski, A. Mycielski, J. Phys. France 39, 87 (1978).

[50] R.R. Gałązka, Semimagnetic semiconductors, in: Proceedings 14th International Conference on the Physics of Semiconductors, Edinburgh 1978, Ed. B.L.H. Wilson, IoP, Bristol 1978, p. 133.

[51] I. Solomon, T. Dietl, D. Kaplan, J. Phys. France 39, 1241 (1978).
[52] M. Dobrowolska, W. Dobrowolski, M. Otto, T. Dietl, R.R. Gałązka, J. Phys. Soc. Jap. 49, Suppl. A, 815 (1980).

[53] A. Mycielski, C. Rigaux, M. Menant, T. Dietl, M. Otto, Solid State Commun. 50, 257 (1984).

[54] T. Dietl, J. Spałek, Phys. Rev. Lett. 48, 355 (1982).

[55] T. Dietl, Post. Fiz. 33, 195 (1982) (in Polish).

[56] J.K. Furdyna, J. Kossut, Diluted Magnetic Semiconductors, Semiconductors and Semimetals, Vol. 25, Academic Press, New York 1988.

[57] T. Dietl, Handbook on Semiconductors, Ed. S. Mahajan, Vol. 3B North-Holland, Amsterdam 1994, p. 1251.

[58] J.A. Gaj, J. Ginter, R.R. Gałązka, Phys. Status Solidi B 89, 655 (1978).

[59] P. Kacman, Semicond. Sci. Technol. 16, R25 (2001).

[60] T. Dietl, C. Śliwa, G. Bauer, H. Pascher, Phys. Rev. B 49, 2230 (1994).

[61] G. Bauer, H. Pascher, W. Zawadzki, Semicond. Sci. Technol. 7, 703 (1992).

[62] T. Story, M. Górska, A. Łusakowski, M. Arciszewska, W. Dobrowolski, E. Grodzicka, Z. Gołacki, R.R. Gałązka, Phys. Rev. Lett. 773447 (1996).

[63] C. Autieri, C. Śliwa, R. Islam, G. Cuono, T. Dietl, Phys. Rev. B 103, 115209 (2021).

[64] C. Benoit à la Guillaume, D. Scalbert, T. Dietl, Phys. Rev. B 46, 9853 (1992).

[65] T. Dietl, Phys. Rev. B 77, 085208 (2008).

[66] W. Pacuski, P. Kossacki, D. Ferrand et al., Phys. Rev. Lett. 100, 037204 (1998).

[67] W. Pacuski, J. Suffczyński, P. Osewski et al., Phys. Rev. B 84, 035214 (2011).

[68] C. Śliwa, T. Dietl, Phys. Rev. B 78, 165205 (2008).

[69] M. Nawrocki, R. Planel, G. Fishman, R.R. Galazka, Phys. Rev. Lett. 46, 735 (1981).

[70] T. Dietl, J. Spałek, Phys. Rev. B 28, 1548 (1983).

[71] T. Dietl, J. Magn. Magn. Mater. 38, 34 (1983).

[72] T. Wojtowicz, S. Koleśnik, I. Miotkowski, J.K. Furdyna, Phys. Rev. Lett. 70, 2317 (1993).

[73] H. Krenn, K. Kaltenegger, T. Dietl, J. Spałek, G. Bauer, Phys. Rev. B 39, 10918 (1989).

[74] T. Dietl, P. Peyla, W. Grieshaber, Y. Merle d'Aubigné, Phys. Rev. Lett. 74, 474 (1995). 
[75] T. Dietl, Phys. Rev. B 91, 125204 (2015).

[76] J. Spałek, A. Lewicki, Z. Tarnawski, J.K. Furdyna, R.R. Gałązka, Z. Obuszko, Phys. Rev. B 33, 3407 (1986).

[77] M. Sawicki, E. Guziewicz, M.I. Łukasiewicz et al., Phys. Rev. B 88, 085204 (2013).

[78] A. Bonanni, T. Dietl, H. Ohno, Dilute Magnetic Materials, in Handbook of Magnetism and Magnetic Materials, Eds. M. Coey, S. Parkin, Springer, 2022.

[79] B. Leclercq, C. Rigaux, A. Mycielski, M. Menant, Phys. Rev. B 47, 6169 (1993).

[80] J. Jaroszyński, J. Wróbel, G. Karczewski, T. Wojtowicz, T. Dietl, Phys. Rev. Lett. 80, 5635 (1998).

[81] T. Story, R.R. Gałązka, R.B. Frankel, P.A. Wolff, Phys. Rev. Lett. 56, 777 (1986).

[82] T. Story, M. Górska, A. Łusakowski, M. Arciszewska, W. Dobrowolski, E. Grodzicka, Z. Gołacki, R.R. Gałązka, Phys. Rev. Lett. 77, 3447 (1996).

[83] T. Suski, J. Igalson, T. Story, J. Magn. Magn. Mater. 66, 325 (1987).

[84] T. Dietl, J. Kossut, Post. Fiz. 34, 322 (1983) (in Polish).

[85] T. Dietl, J. Antoszewski, L. Świerkowski, Physica $B+C$ 117-118, 491 (1983).

[86] T. Wojtowicz, A. Mycielski, Physica $B+C$ 117-118, 476 (1983).

[87] Y. Ono, J. Kossut, J. Phys. Soc. Japan 53, 1128 (1984).

[88] M. Sawicki, T. Dietl, J. Kossut, J. Igalson, T. Wojtowicz, W. Plesiewicz, Phys. Rev. Lett. 56, 508 (1986).

[89] T. Andrearczyk, J. Jaroszyński, G. Grabecki, T. Dietl, T. Fukumura, M. Kawasaki, Phys. Rev. B 72, 121309(R) (2005).

[90] T. Dietl, T. Andrearczyk, A. Lipińska, M. Kiecana, Maureen Tay, Yihong Wu, Phys. Rev. B 76, 155312 (2007).

[91] J. Jaroszyński, T. Andrearczyk, G. Karczewski, J. Wróbel, T. Wojtowicz, D. Popović, T. Dietl, Phys. Rev. B 76, 045322 (2007).

[92] R. Adhikari, W. Stefanowicz, B. Faina, G. Capuzzo, M. Sawicki, T. Dietl, A. Bonanni, Phys. Rev. B 91, 205204 (2015).

[93] T. Dietl, L. Świerkowski, J. Jaroszyński, M. Sawicki, T. Wojtowicz, Phys. Scr. T14, 29 (1986).

[94] P. Głód, T. Dietl, M. Sawicki, I. Miotkowski, Phys. B: Condensed Matter 194-196, 995 (1994).
[95] T. Dietl, J. Phys. Soc. Jpn. 77, 031005 (2008).

[96] T. Wojtowicz, T. Dietl, M. Sawicki, W. Plesiewicz, J. Jaroszyński, Phys. Rev. Lett. 56, 2419 (1986).

[97] T. Wojtowicz, M. Sawicki, J. Jaroszyński, T. Dietl, W. Plesiewicz, Phys. B: Condens. Matter 155, 357 (1989).

[98] J. Jaroszyński, T. Dietl, Physica B 177, 469 (1992).

[99] W. Stefanowicz, R. Adhikari, T. Andrearczyk, B. Faina, M. Sawicki, J.A. Majewski, T. Dietl, A. Bonanni, Phys. Rev. B 89, 205201 (2014).

[100] T. Dietl, Japan. J. Appl. Phys. 26, 1907 (1987).

[101] T. Dietl, M. Sawicki, J. Jaroszyński, T. Wojtowicz, W. Plesiewicz, A. Lenard, Semimagnetic Semiconductors Near the Metal-Insulator Transition, in: 19th International Conference on the Physics of Semiconductors, Warsaw 1988, Ed. W. Zawadzki IF PAN, Warsaw 1988, p. 1189.

[102] T. Dietl, M. Sawicki, M. Dahl, D. Heiman, E.D. Isaacs, M.J. Graf, S.I. Gubarev, D.L. Alov, Phys. Rev. B 43, 3154 (1991).

[103] T. Dietl, Post. Fiz. 48, 159 (1997) (in Polish).

[104] T. Omiya, F. Matsukura, T. Dietl, Y. Ohno, T. Sakon, M. Motokawa, H. Ohno, Phys. E: Low-Dimens. Syst. Nanostruct. 7, 976 (2000).

[105] T. Dietl, M. Sawicki, Mn-Based Ferromagnetic Semiconductors, in: Quantum Sensing: Evolution and Revolution from Past to Future, Vol. 4999, Eds. M. Razeghi, G.J. Brown, International Society for Optics and Photonics, SPIE, 2003 p. 19.

[106] F. Matsukura, M. Sawicki, T. Dietl, D. Chiba, H. Ohno, Phys. E: Low-Dimens. Syst. Nanostruct. 21, 1032 (2004).

[107] A. Kazakov, W. Brzezicki, T. Hyart et al., arXiv:2002.07622, 2020.

[108] W. Walukiewicz, J. Phys. C: Solid State Phys. 9, 1945 (1976).

[109] T. Dietl, J. Dubowski, W. Szymańska, in: Proc. 14th Int. Conf. on the Physics of Semiconductors, Edinburgh 1978, Ed. B.L.H. Wilson, IoP, Bristol 1978, p. 245.

[110] Z. Wilamowski, K. Świątek, T. Dietl, J. Kossut, Solid State Commun. 74, 833 (1990).

[111] A. Mycielski, J. Appl. Phys. 63, 3279 (1988).

[112] J. Kossut, W. Dobrowolski, Z. Wilamowski, T. Dietl, K. Swiatek, Semicond. Sci. Techn. 5, S260 (1990). 
[113] T. Suski, P. Wiśniewski, E. LitwinStaszewska, J. Kossut, Z. Wilamowski, T. Dietl, K. Swiatek, K. Ploog, J. Knecht, Semicond. Sci. Techn. 5, 261 (1990).

[114] J. Wróbel, T. Dietl, A. Łusakowski, G. Grabecki, K. Fronc, R. Hey, K.H. Ploog, H. Shtrikman, Phys. Rev. Lett. 93, 246601 (2004).

[115] T. Dietl, Acta Phys. Pol. A 69, 817 (1986).

[116] T. Dietl, G. Grabecki, J. Jaroszynski, Semicond. Sci. Technol. 8, S141 (1993).

[117] G. Grabecki, T. Dietl, J. Kossut, W. Zawadzki, Surf. Sci. 142, 588 (1984).

[118] G. Grabecki, T. Dietl, P. Sobkowicz, J. Kossut, W. Zawadzki, Appl. Phys. Lett. 45, 1214 (1984).

[119] G. Grabecki, T. Suski, T. Dietl, T. Skośkiewicz, M. Gliński, in: High Magnetic Fields in Semiconductor Physics, Ed. G. Landwehr, Springer Berlin Heidelberg, Berlin 1987, p. 127.

[120] G. Grabecki, A. Wittlin, T. Dietl, P.A.A. Teunissen, S.A.J. Wiegers, J.A.A.J. Perenboom, Semicond. Sci. Techn. 8, S95 (1993).

[121] M.A. Herman, H. Sitter, Molecular Beam Epitaxy: Fundamentals and Current Status, Springer, Berlin 1989.

[122] G. Grabecki, A. Lenard, W. Plesiewicz et al., Acta Phys. Pol. A 80, 307 (1991).

[123] J. Jaroszyński, J. Wróbel, M. Sawicki et al., Phys. Rev. Lett. 75, 3170 (1995).

[124] the Physics of Diluted Magnetic Semiconductors, Eds. J. Kossut, J.A. Gaj, Springer, Berlin 2010.

[125] A. Kazakov, T. Wojtowicz, 2d Electron Gas in Chalcogenide Multilayers, in: Chalcogenide, Woodhead Publishing Series in Electronic and Optical Materials, Eds. Xinyu Liu, Sanghoon Lee, J.K. Furdyna, Tengfei Luo, Yong-Hang Zhang, Woodhead Publishing, 2020, p. 189.

[126] M. Goryca, T. Kazimierczuk, M. Nawrocki, A. Golnik, J.A. Gaj, P. Kossacki, P. Wojnar, G. Karczewski, Phys. Rev. Lett. 103, 087401 (2009).

[127] P. Wojnar, E. Janik, L.T. Baczewski et al., Nano Lett. 12, 3404 (2012).

[128] J. Jaroszyński, T. Andrearczyk, G. Karczewski et al., Phys. Rev. Lett. 89, 266802 (2002).

[129] C. Betthausen, T. Dollinger, H. Saarikoski, V. Kolkovsky, G. Karczewski, T. Wojtowicz, K. Richter, D. Weiss, Science 337, 324 (2012).
[130] B.A. Piot, J. Kunc, M. Potemski, D.K. Maude, C. Betthausen, A. Vogl, D. Weiss, G. Karczewski, T. Wojtowicz, Phys. Rev. B 82, 081307 (2010).

[131] C. Betthausen, P. Giudici, A. Iankilevitch et al., Phys. Rev. B 90, 115302 (2014).

[132] A. Kazakov, G. Simion, Y. Lyanda-Geller, V. Kolkovsky, Z. Adamus, G. Karczewski, T. Wojtowicz, L.P. Rokhinson, Phys. Rev. Lett. 119, 046803 (2017).

[133] G. Simion, A. Kazakov, L.P. Rokhinson, T. Wojtowicz, Y.B. Lyanda-Geller, Phys. Rev. B 97, 245107 (2018).

[134] T. Dietl, A. Haury, Y. Merle d'Aubigné, Phys. Rev. B 55, R3347 (1997).

[135] T. Dietl, H. Ohno, F. Matsukura, J. Cibert, D. Ferrand, Science 287, 1019 (2000).

[136] T. Dietl, H. Ohno, F. Matsukura, Phys. Rev. B 63, 195205 (2001).

[137] T. Dietl, J. Cibert, D. Ferrand, Y. Merle d'Aubigné, Mater. Sci. Engin. B 63, 103 (1999).

[138] A. Haury, A. Wasiela, A. Arnoult, J. Cibert, S. Tatarenko, T. Dietl, Y. Merle d'Aubigné, Phys. Rev. Lett. 79, 511 (1997).

[139] H. Boukari, P. Kossacki, M. Bertolini, D. Ferrand, J. Cibert, S. Tatarenko, A. Wasiela, J.A. Gaj, T. Dietl, Phys. Rev. Lett. 88, 207204 (2002).

[140] D. Kechrakos, N. Papanikolaou, K.N. Trohidou, T. Dietl, Phys. Rev. Lett. 94, 127201 (2005).

[141] A. Lipińska, C. Simserides, K.N. Trohidou, M. Goryca, P. Kossacki, A. Majhofer, T. Dietl, Phys. Rev. B 79, 235322 (2009).

[142] D. Ferrand, J. Cibert, C. Bourgognon et al., J. Crys. Growth 214-215, 387 (2000).

[143] D. Ferrand, J. Cibert, A. Wasiela et al., Phys. Rev. B 63, 085201 (2001).

[144] Le Van Khoi, M. Sawicki, K. Dybko, V. Domukhovski, T. Story, T. Dietl, A. Jędrzejczak, J. Kossut, R.R. Gałązka, Phys. Status Solidi B 229, 53 (2002).

[145] H. Kępa, Van Khoi Le, C.M. Brown, M. Sawicki, J.K. Furdyna, T.M. Giebułtowicz, T. Dietl, Phys. Rev. Lett. 91, 087205 (2003).

[146] T. Andrearczyk, J. Jaroszyński, M. Sawicki et al., in: Proc. 25th Int. Conf. on Physics of Semiconductors, Osaka (Japan) 2000, Eds. N. Miura, T. Ando, Springer, Berlin 2001, p. 235.

[147] T. Dietl, Nat. Mater. 9, 965 (2010). 
[148] M. Csontos, G. Mihály, B. Jankó, T. Wojtowicz, X. Liu, J.K. Furdyna, Nat. Mater. 4, 447 (2005).

[149] M. Gryglas-Borysiewicz, A. Kwiatkowski, P. Juszyński et al., Phys. Rev. B 101, 054413 (2020).

[150] A. Werpachowska, T. Dietl, Phys. Rev. B 82, 085204 (2010).

[151] C. Śliwa, T. Dietl, Phys. Rev. B 83, 245210 (2011).

[152] A. Łusakowski, P. Bogusławski, T. Story J. Phys.: Condens. Matter 27, 226002 (2015).

[153] T. Dietl, J. König, A.H. MacDonald, Phys. Rev. B 64, 241201 (2001).

[154] M. Sawicki, F. Matsukura, A. Idziaszek, T. Dietl, G.M. Schott, C. Rüester, C. Gould, G. Karczewski, G. Schmidt, L.W. Molenkamp, Phys. Rev. B 70, 245325 (2004).

[155] M. Sawicki, K.-Y. Wang, K.W. Edmonds et al., Phys. Rev. B 71, 121302 (2005).

[156] K.-Y. Wang, M. Sawicki, K.W. Edmonds, R.P. Campion, S. Maat, C.T. Foxon, B.L. Gallagher, T. Dietl, Phys. Rev. Lett. 95, 217204 (2005).

[157] M. Sawicki, D. Chiba, A. Korbecka, Yu. Nishitani, J.A. Majewski, F. Matsukura, T. Dietl, H. Ohno, Nat. Phys. 6, 22 (2010).

[158] M. Sawicki, O. Proselkov, C. Sliwa, P. Aleshkevych, J.Z. Domagala, J. Sadowski, T. Dietl, Phys. Rev. B 97, 184403 (2018).

[159] W. Stefanowicz, C. Śliwa, P. Aleshkevych, T. Dietl, M. Döppe, U. Wurstbauer, W. Wegscheider, D. Weiss, M. Sawicki, Phys. Rev. B 81, 155203 (2010).

[160] D. Chiba, M. Sawicki, Y. Nishitani, Y. Nakatani, F. Matsukura, H. Ohno, Nature 455, 515 (2008).

[161] Y. Nishitani, D. Chiba, M. Endo, M. Sawicki, F. Matsukura, T. Dietl, H. Ohno, Phys. Rev. B 81, 045208 (2010).

[162] M. Yamanouchi, D. Chiba, F. Matsukura, T. Dietl, H. Ohno, Phys. Rev. Lett. 96, 096601 (2006).

[163] P. Van Dorpe, W. Van Roy, J. De Boeck, G. Borghs, P. Sankowski, P. Kacman, J.A. Majewski, T. Dietl, Phys. Rev. B 72, 205322 (2005).

[164] P. Sankowski, P. Kacman, J.A. Majewski, T. Dietl, Phys. Rev. B 75, 045306 (2007).

[165] T. Dietl, Acta Phys. Pol. A 100, 139 (2001).

[166] T. Dietl, Semicond. Sci. Technol. 17, 377 (2002).
[167] C. Rüester, T. Borzenko, C. Gould et al., Phys. Rev. Lett. 91, 216602 (2003).

[168] M.J. Grzybowski, P. Wadley, K.W. Edmonds et al., Phys. Rev. Lett. 118, 057701 (2017).

[169] M.J. Grzybowski, P. Wadley, K.W. Edmonds, R.P. Campion, K. Dybko, M. Majewicz, B.L. Gallagher, M. Sawicki, T. Dietl, AIP Adv. 9, 115101 (2019).

[170] J. Sadowski, H. Mariette, A. Wasiela, R. André, Y. Merle d'Aubigné, T. Dietl, Phys. Rev. B 56, R1664 (1997).

[171] Y. Merle d'Aubigné, A. Wasiela, H. Mariette, T. Dietl, Phys. Rev. B 54, 14003 (1996).

[172] T. Jungwirth, J. Sinova, A.H. MacDonald et al., Phys. Rev. B 76, 125206 (2007).

[173] D. Chiba, A. Werpachowska, M. Endo, Y. Nishitani, F. Matsukura, T. Dietl, H. Ohno, Phys. Rev. Lett. 104, 106601 (2010).

[174] T. Dietl, F. Matsukura, H. Ohno, Phys. Rev. B 66, 033203 (2002).

[175] W. Stefanowicz, D. Sztenkiel, B. Faina et al., Phys. Rev. B 81, 235210 (2010).

[176] A. Bonanni, M. Sawicki, T. Devillers et al., Phys. Rev. B 84, 035206 (2011).

[177] M. Sawicki, T. Devillers, S. Gałęski et al., Phys. Rev. B 85, 205204 (2012).

[178] G. Kunert, S. Dobkowska, Tian Li et al., Appl. Phys. Lett. 101, 022413 (2012).

[179] S. Stefanowicz, G. Kunert, C. Simserides et al., Phys. Rev. B 88, 081201 (2013).

[180] J. Blinowski, P. Kacman, J.A. Majewski, Phys. Rev. B 53, 9524 (1996).

[181] D. Sztenkiel, M. Foltyn, G.P. Mazur et al., Nat. Commun. 7, 13232 (2016).

[182] J. Ginter, J. Kossut, L. Świerkowski, Phys. Status Solidi B 96, 735 (1979).

[183] T. Dietl, Nat. Mater. 2, 646 (2003).

[184] G. Karczewski, M. Sawicki, V. Ivanov, C. Rüester, G. Grabecki, F. Matsukura, L.W. Molenkamp, T. Dietl, J. Supercond. Nov. Magn. 16, 55 (2003).

[185] T. Dietl, J. Magn. Magn. Mater. 290291, 14 (2005).

[186] A. Bonanni, M. Kiecana, C. Simbrunner et al., Phys. Rev. B 75, 125210 (2007).

[187] A. Bonanni, A. Navarro-Quezada, Tian Li et al., Phys. Rev. Lett. 101, 135502 (2008).

[188] S. Kuroda, N. Nishizawa, K. Takita, M. Mitome, Y. Bando, K. Osuch, T. Dietl, Nature Mat. 6, 440 (2007).

[189] M. Sawicki, W. Stefanowicz, A. Ney, Semicond. Sci. Technol. 26, 064006 (2011). 
[190] K. Gas, G. Kunert, P. Dluzewski, R. Jakiela, D. Hommel, M. Sawicki, J. Alloy. Comp. 868, 159119 (2021).

[191] T. Dietl, J. Appl. Phys. 103, $07 \mathrm{D} 111$ (2008).

[192] A. Bonanni, T. Dietl, Chem. Soc. Rev. 39, 528 (2010).

[193] T. Dietl, Nat. Mater. 5, 673 (2006).

[194] T. Devillers, M. Rovezzi, N. Gonzalez Szwacki et al., Sci. Rep. 2, 722 (2012).

[195] J. Blinowski, P. Kacman, Phys. Rev. B 67, 121204 (2003).

[196] K.W. Edmonds, P. Bogusławski, K.Y. Wang et al., Phys. Rev. Lett. 92, 037201 (2004).

[197] N. Gonzalez Szwacki, J.A. Majewski, T. Dietl, Phys. Rev. B 83, 184417 (2011).

[198] M. Birowska, C. Śliwa, J.A. Majewski, T. Dietl, Phys. Rev. Lett. 108, 237203 (2012).
[199] Y. Yuan, R. Hübner, M. Birowska et al., Phys. Rev. Materials 2, 114601 (2018).

[200] A. Navarro-Quezada, K. Gas, T. Truglas et al., Materials 13, 3294 (2020).

[201] N. Pournaghavi, M.F. Islam, R. Islam, C. Autieri, T. Dietl, C.M. Canali, arXiv:2101.06259, 2021.

[202] A. Lenard, W. Plesiewicz, M. Sawicki, T. Dietl, Cryogenics 34, 429 (1994).

[203] T. Dietl, Acta Phys. Pol. A 73, 793 (1988).

[204] A. Lenard, T. Dietl, M. Sawicki et al., J. Low Temp. Phys. 80, 15 (1990).

[205] S.D. Darchuk, L.A. Korovina, F.F. Sizov, T. Dietl, S. Kolesnik, M. Sawicki, Semiconductors 32, 700 (1998).

[206] G. Grabecki, K.A. Kolwas, J. Wróbel et al., J. Appl. Phys. 108, 053714 (2010). 